\title{
Equatorial inertial-parametric instability of zonally symmetric oscillating shear flows
}

\author{
By MARC D'ORGEVILLE AND BACH LIEN HUA \\ Laboratoire de Physique des Océans, IFREMER, BP 70, 29280 Plouzané, France
}

(Received 5 June 2004 and in revised form 6 December 2004)

This study revisits the problem of the zonally symmetric instability on the equatorial $\beta$-plane. Rather than treating the classical problem of a steady basic flow, it treats a sequence of problems of increasing complexity in which the basic flow is oscillatory in time with a frequency $\omega_{0}$.

First, for the case of a homogeneous fluid, a time-oscillating barotropic shear forcing may excite a subharmonic parametric resonance of inertial oscillations. Because of the continuous distribution of inertial oscillation frequencies, this resonance occurs at critical inertial latitudes $y_{c}$ such that $\beta y_{c}= \pm \omega_{0} / 2$. Next the effects of stratification, characterized by Brunt-Väisälä frequency $N$, are taken into account. It is shown analytically (in the asymptotic limit of a weak shear) that the forced temporal oscillation leads to an inertial-parametric instability, when a resonance condition between the basic flow frequency and the sum of two inertio-gravity free-mode frequencies is met. This inertial-parametric instability has a well-defined inviscid vertical scale selection favouring the high-vertical mode $m_{c} \sim 7.45 m_{0}$, where $m_{0}=\beta N / \omega_{0}^{2}$ is the equatorial vertical mode characteristic of frequency $\omega_{0}$. The viscous critical shear of inertial-parametric instability is lower than the steady inertial instability one.

Finally, this type of setting naturally arises when the basic flow is considered to be an equatorial wave, so the problem is recast with the nonlinear adjustment of the vertically sinusoidal basic state of a zonally symmetric mixed Rossby-gravity (MRG) wave. Initial-value numerical simulations show that the same inertial-parametric instability exists leading to a resonant subharmonic excitation of free modes with vertical scales 7 and 8 times smaller than the basic-state wave. A simplified dynamical model of the instability is introduced, demonstrating that the oscillatory nature of the shear with height for the MRG wave necessarily implies a resonance between distinct vertical modes, the most unstable ones being modes 7 and 8 for a large enough Froude number of the MRG wave. The nonlinear action of the instability is described in terms of angular momentum and potential vorticity changes: a significant mixing due to the breaking of the excited high vertical modes creates a vertically averaged westward flow at the equator and extra-equatorial eastward flows. The ideas exposed may play a part in explaining layering phenomena and the latitudinal structure of the zonal flow in the equatorial oceans below the thermocline.

\section{Introduction}

The deep equatorial ocean offers many examples of phenomena where a substantial latitudinal homogenization of angular momentum appears as the background largevertical-scale ambient state (Hua, Moore \& Le Gentil 1997; Bourlès et al. 2003), upon which other smaller vertical scale phenomena such as Equatorial Deep Jets (Firing 
1987; Gouriou et al. 2001) or extended density layering (d'Orgeville et al. 2004) appear to be superposed. The issue of angular momentum homogenization has been mostly studied in the context of the Earth's and other planets' atmospheres (e.g. Held \& Hou 1980) in a zonally symmetric steady context. Such work brings out the characteristic spatial scales of homogenization of angular momentum for a given planet's atmosphere as being related to its equatorial radius of deformation, a quantity governed by the magnitudes of stratification and rotation, which will characterize the meridional extent of the meridional Hadley cells that are set up in the presence of an equator-pole temperature difference. Such cellular motions in the meridional plane are indeed likely to mix laterally angular momentum and a related quantity, namely potential vorticity, in the general case of a stratified and rotating fluid. Such concepts have been applied in an oceanic context by Marin, Hua \& Wacongne (2000), Hua, Marin \& Schopp (2003) and Marin, Schopp \& Hua (2003) to explain the temperature and potential vorticity homogenization observed just below the main thermocline in both the Pacific and Atlantic equatorial oceans.

A plausible underlying mechanism which could be responsible for this potential vorticity homogenization could be inertial instability, for which a necessary condition for a zonally symmetric equatorial steady flow is that potential vorticity $(P V)$ is of opposite sign to the Coriolis parameter $f$ in a given hemisphere, i.e. $f P V<0$ (Hoskins 1974; Dunkerton 1981; Stevens 1983). Recent studies of nonlinear regimes of equatorial inertial instability are the work of Hua et al. (1997) and Griffiths $(2003 a, b)$, and more generally for nonlinear symmetric instability $(\mathrm{Mu}$, Shepherd \& Swanson 1996).

However, for the deep equatorial ocean, there is no known source of stationary shear which can create regions of $f P V<0$, but, on the other hand, a ubiquituous feature is the presence over the entire fluid depth of periodic signals, usually identified as equatorial free waves (e.g. Gill 1982). Such temporal variability due to wave activity could induce periodic shear and this is precisely the mechanism we want to investigate here.

A periodic forcing of equations which can sustain free oscillating solutions is well-known to be propitious to parametric resonances, an archetype being provided by Mathieu's equation which can lead to subharmonic resonances at frequencies lower than the forcing frequency. There has been a recent renewal of interest in the study of parametric instability in geophysical flows, either for stratified flows for the parametric excitation of internal waves (Benielli \& Sommeria 1998; Staquet \& Sommeria 2002), or for rotating shear flows (e.g. Poulin, Flierl \& Pedlosky 2003, and references therein) or for both stratified and rotating flows for the baroclinic instability problem (Pedlosky \& Thomson 2003; Pavec, Carton \& Swaters 2005). Parametric instability has also been identified as the generic mechanism underlying elliptic instability (e.g. McWilliams \& Yavneh 1998; Kerswell 2002) and also underlying the layering commonly seen in turbulent stratified flows (Leblanc 2003). In the same vein, Majda \& Shefter $(1998 a, b)$ have invoked the parametric destabilization of internal waves to be at the origin of numerous layering observations in the ocean.

All these points motivate the main questions which are addressed in the present work:

(i) In view of the specific property of equatorial regions that a continuous range of inertial oscillation frequencies exists, can such motions be parametrically excited?

(ii) Can large-vertical scale equatorial free waves lead to parametric resonances and impact angular momentum distribution? 
A necessary ingredient for equatorial inertial instability being the existence of lateral shear, which, for a zonal flow, will cause angular momentum to reach its maximum away from the equator, these two questions could be addressed by considering the simplest generalization of the equatorial steady inertial instability problem which is the case of an equatorial oscillating shear flow.

This is precisely what is done in the present work, in treating a sequence of problems of increasing complexity in which the basic flow is oscillatory in time with a frequency $\omega_{0}$. The idealized case of a barotropic oscillating shear forcing is investigated for an homogeneous fluid in $\S 2$, then for a stratified fluid in $\S 3$. Results of previous sections about the main characteristics of inertial-parametric instability are used in $\S 4$ for the study of the nonlinear adjustment of the simplest zonally symmetric equatorial free wave with such lateral shear, the mixed Rossby-gravity (MRG) wave. The final state of the nonlinear adjustment is described in terms of angular momentum and potential vorticity changes in $\S 5$, and finally $\S 6$ summarizes our findings and discusses their importance for geophysical flows.

\section{Homogeneous fluid case: resonance of inertial free motions at critical inertial latitudes}

Throughout this paper, $\widetilde{R}$ corresponds to a basic-state variable, $r^{\prime}$ to its perturbation and $r$ the total field such that $r=\widetilde{R}+r^{\prime}$. The terms $u, v$ and $w$ are, respectively, the zonal, meridional and vertical velocities. The terms $x, y, z$ and $t$ denote, respectively, the zonal, meridional, vertical and time coordinates. We shall assume zonally symmetric motions $(\partial / \partial x=0)$, both for the basic-state variables and for perturbations.

In this section and the following one, we consider the idealized forced case of an oscillating strictly zonal shear flow in geostrophic balance

$$
\begin{gathered}
\widetilde{U}=\gamma y \cos \left(\omega_{0} t\right), \quad \widetilde{V}=0, \\
f \widetilde{U}=-\frac{1}{\rho_{0}} \frac{\partial \tilde{p}}{\partial y}, \\
\frac{\partial \widetilde{U}}{\partial t}=F(y, t),
\end{gathered}
$$

where $\gamma$ is the shear, $\omega_{0}$ the pulsation, $f=2 \Omega \sin \phi$ the Coriolis parameter $(\Omega$ denoting the Earth's rotation rate and $\phi$ the latitude), $\rho_{0}$ the reference density of the Boussinesq approximation, $\widetilde{p}$ is the barotropic pressure and $F$ is a prescribed external forcing.

Such restrictive conditions for the forcing set-up will be relaxed in $\S \S 4$ and 5 concerning the stability of the free equatorial mixed Rossby-gravity wave. The purpose of this set-up is to characterize analytically the spatial meridional and vertical scales of the problem that will be useful for the last sections of the paper.

\section{1. $f$-plane vs. equatorial $\beta$-plane: critical latitude selection}

In an $f$-plane framework $\left(f=f_{0}\right)$, the horizontal trajectory of a fluid parcel which is only subject to the Coriolis force is an inertial circular path, which is commonly observed in geophysical flows. Such a free oscillation should be susceptible to being parametrically excited for an appropriate periodic forcing.

In order, as a first step, not to have to consider effects due to stratification, we first restrict ourselves to the case of a homogeneous fluid forced by the basic state (2.1)-(2.3) and, moreover, to the case of free inertial oscillations (for which pressure 
gradient forces are dropped: their effects will be taken into account in the case of a stratified fluid, $\S 3$ ).

Denoting the total derivative as $\mathrm{d} / \mathrm{d} t=\partial / \partial t+v^{\prime} \partial / \partial y+w^{\prime} \partial / \partial z,(2.1)-(2.3)$ for the basic state imply that velocity perturbations $\left(u^{\prime}, v^{\prime}\right)$ obey

$$
\begin{gathered}
(\mathrm{d} / \mathrm{d} t) u^{\prime}-\left(f_{0}-\gamma \cos \left(\omega_{0} t\right)\right) v^{\prime}=0, \\
(\mathrm{~d} / \mathrm{d} t) v^{\prime}+f_{0} u^{\prime}=0 .
\end{gathered}
$$

Eliminating $u^{\prime}$ in these equations yields

$$
\frac{\mathrm{d}^{2}}{\mathrm{~d} t^{2}} v^{\prime}+\left(f_{0}^{2}-f_{0} \gamma \cos \left(\omega_{0} t\right)\right) v^{\prime}=0
$$

which is the well-known Mathieu's equation (e.g. Nayfeh \& Mook 1979).

The flow will exhibit parametric resonances depending on the values of the oscillating shear frequency $\omega_{0}$ and on its strength $\gamma$. The largest resonance is subharmonic and occurs for $f_{0}=\omega_{0} / 2$. Other resonances in Mathieu's equation are also possible for $f_{0}=p \omega_{0} / 2$, with integer values of $p \geqslant 2$, but correspond to weaker growth rates.

For the $f$-plane case, the inertial frequency is unique and the excitation frequency spectrum which can induce parametric resonances is discrete. In contrast, for an equatorial $\beta$-plane $(f=\beta y)$, there is a continuous inertial frequency distribution which should allow the possibility of parametric resonance for any value of the excitation frequency. This is the set-up that we shall consider next.

Latitudinal displacements $y$ of a fluid parcel with velocity perturbations $\left(u^{\prime}, v^{\prime}\right)$ are such that

$$
\begin{gathered}
\frac{\mathrm{d}}{\mathrm{d} t} y=v^{\prime}, \\
\frac{\mathrm{d}}{\mathrm{d} t} v^{\prime}+\beta y u^{\prime}=0, \\
\frac{\mathrm{d}}{\mathrm{d} t} u^{\prime}-\left(\beta y-\gamma \cos \left(\omega_{0} t\right)\right) v^{\prime}=0 .
\end{gathered}
$$

Introducing non-dimensional quantities $y=\left(\omega_{0} / \beta\right) z_{1}, v^{\prime}=\left(\omega_{0}^{2} / \beta\right) z_{2}, u^{\prime}=\left(\omega_{0}^{2} / \beta\right) z_{3}$, $\gamma=\omega_{0} \gamma^{*}$ and $t=t^{*} / \omega_{0}$ (hereinafter asterisks are dropped) leads to

$$
\frac{\mathrm{d}}{\mathrm{d} t}\left(\begin{array}{l}
z_{1} \\
z_{2} \\
z_{3}
\end{array}\right)=\left(\begin{array}{c}
z_{2} \\
-z_{1} z_{3} \\
z_{1} z_{2}-\gamma \cos (t) z_{2}
\end{array}\right),
$$

which corresponds to a nonlinear system which is first-order in time. Equilibrium points are $\left(z_{1}, z_{2}, z_{3}\right)=\left(0,0, u_{i}\right)$ and $\left(z_{1}, z_{2}, z_{3}\right)=\left(y_{i}, 0,0\right)$. A stability analysis can be performed in the vicinity of these equilibrium points.

(i) $\left(z_{1}, z_{2}, z_{3}\right)=\left(\delta y, \delta v, u_{i}+\delta u\right)$ : a linear stability analysis of the above system yields an equation for the evolution of $\delta y$ at zeroth order

$$
\frac{\mathrm{d}^{2}}{\mathrm{~d} t^{2}} \delta y+u_{i} \delta y=0
$$

which is stable for $u_{i}>0$ and unstable for $u_{i}<0 .\left(0,0, u_{i}\right)$ corresponds to a fluid parcel initially located at the equator with no meridional velocity. For $u_{i}>0$, the fluid parcel corresponds to a maximum of angular momentum located at the equator and thus is inertially stable, whereas for $u_{i}<0$ it will be inertially unstable. Note that such instability is independent of the temporal forcing. 

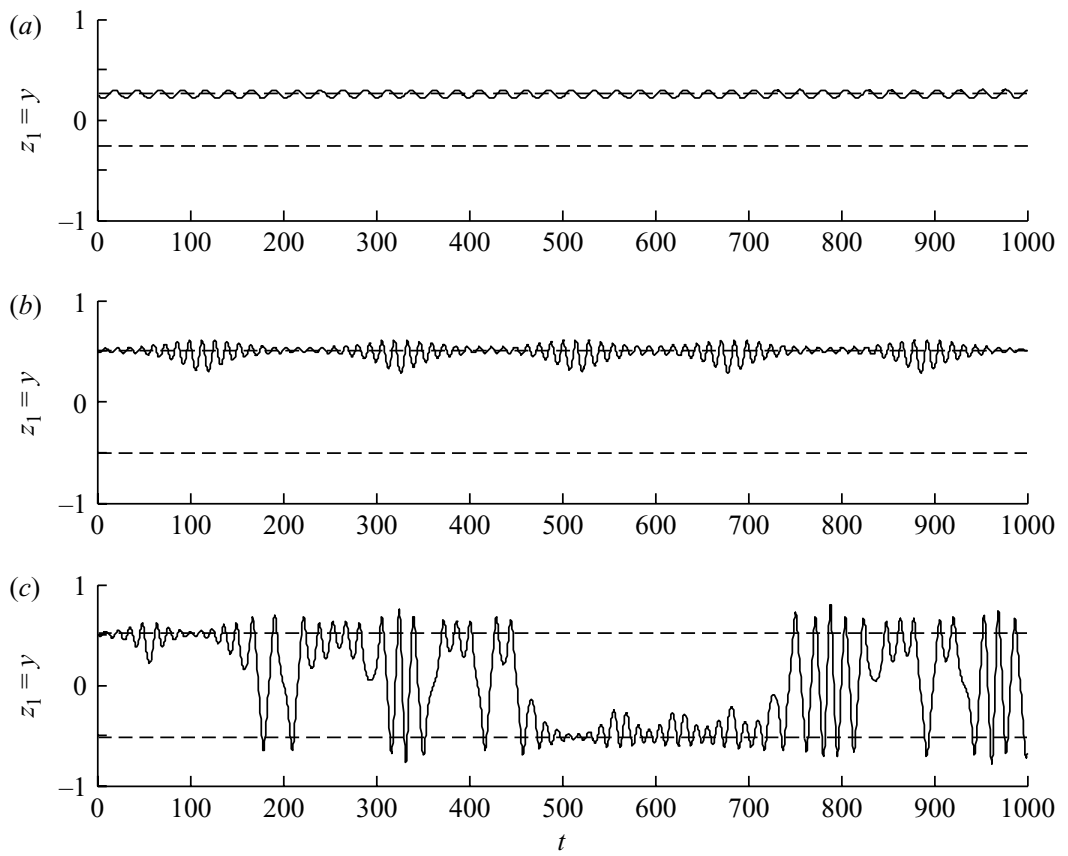

FiguRE 1. Direct numerical integration of (2.4) with $\delta y_{i}=0.02, \delta v_{i}=0.01$ for the homogeneous case. (a) $\gamma=0.1$ and $y_{i}=0.25$ non-resonant latitude. $(b) \gamma=0.1$ and $y_{i}=0.5$ resonant latitude. (c) $\gamma=0.2$ and $y_{i}=0.5$ resonant latitude. The axes are time $t$ (abcissa) and latitudinal position $z_{1}=y$ (ordinate).

(ii) $\left(z_{1}, z_{2}, z_{3}\right)=\left(y_{i}+\delta y, \delta v, \delta u\right)$ : a linear stability analysis yields a Mathieu-type equation for the evolution of $\delta v$ at zeroth order

$$
\frac{\mathrm{d}^{2}}{\mathrm{~d} t^{2}} \delta v+\left(y_{i}^{2}-y_{i} \gamma \cos (t)\right) \delta v=0 .
$$

Possible resonances thus depend on the latitudinal position of the equilibrium point $y_{i}$ and on the shear strength $\gamma$, the strongest resonance occurring at $y_{i}= \pm 1 / 2$.

In contrast to the $f$-plane case which could resonate or not, depending on the value of the excitation frequency $\omega_{0}$, in the equatorial $\beta$-plane case, for a given value of the excitation frequency, there will always exist latitudes $y_{i}$ which will resonate inertially. Such selection of resonant latitudes by an oscillating shear flow can be illustrated by a direct numerical integration of the fully nonlinear system (2.4).

Figure 1 presents the temporal evolution of the latitudinal position of a fluid parcel, initially at $y_{i}+\delta y_{i}$, which is given a weak initial impulse $\delta v_{i}$. Initial conditions for integration of system (2.4) are thus $z_{i}=\left(y_{i}+\delta y_{i}, \delta v_{i}, 0\right)$. Figure 1(a) displays a simple inertial oscillation about latitude $y_{i}$ with a frequency close to $y_{i}$ (dimensionally corresponding to $\beta y_{i}$ ). On the contrary, figure 1(b) illustrates a resonance about $y_{i}=0.5$ : inertial oscillations are parametrically amplified as predicted by (2.5), until nonlinear terms come into play to restabilize the motion. However, for increased values of $\gamma$ (figure $1 c$ ), the instability enables parcel motions across the equator: the parcel trajectory between the two critical latitudes $y_{i}= \pm 0.5$ seems to become chaotic.

We have shown in the case of a homogeneous fluid the possibility of an equatorial parametric instability induced by an oscillating zonal shear flow: a Mathieu-like resonance of inertial oscillations appears at the critical latitude $y_{c}$ where the local 
inertial frequency $f=\beta y$ is half the excitation frequency $2 f \sim \omega_{0} \Leftrightarrow y_{c} \sim \omega_{0} / 2 \beta$. In the deep equatorial ocean, a typical time scale of the observed variability is 15 days, which corresponds to critical latitudes around $\pm 1^{\circ}$. Because of the latitudinal limit for the validity of the equatorial $\beta$-plane approximation, a realistic upper bound for $\omega_{0}$ would be $2 \pi /$ day for critical latitudes around $\pm 15^{\circ}$.

For this homogeneous fluid study, the only restoring force at the origin of free oscillations is the Coriolis force due to the Earth's rotation. In the next section, we shall consider the same problem, but for a stratified fluid, where baroclinic pressure gradients can modify the fluid adjustment, but for which a subharmonic inertial resonance is still the main driving mechanism.

\section{Stratified fluid case: subharmonic resonance of inertio-gravity waves}

We next take into account the influence of stratification and denote the background Brunt-Väisälä frequency $N$, with $N^{2}=-\left(g / \rho_{0}\right)(\mathrm{d} \bar{\rho} / \mathrm{d} z)$, where $g$ is gravity and $\bar{\rho}(z)$ is the background mean density at a given depth of the fluid at rest.

Zonally symmetric conditions imply the continuity equation $\partial v^{\prime} / \partial y+\partial w^{\prime} / \partial z=0$, and we introduce a meridional streamfunction $\psi^{\prime}$ such that $v^{\prime}=-\partial \psi^{\prime} / \partial z, w^{\prime}=\partial \psi^{\prime} / \partial y$. Thus, the equations of motion are those of zonal velocity perturbation $u^{\prime}$, zonal vorticity $\xi^{\prime}=\nabla^{2} \psi^{\prime}$ and rescaled density anomaly $b^{\prime}=\left(g / \rho_{0}\right) \rho^{\prime}$

$$
\begin{gathered}
\frac{\partial u^{\prime}}{\partial t}+J\left(\psi^{\prime}, u^{\prime}\right)+\left(\beta y-\gamma \cos \left(\omega_{0} t\right)\right) \frac{\partial \psi^{\prime}}{\partial z}=D_{u^{\prime}}, \\
\frac{\partial \xi^{\prime}}{\partial t}+J\left(\psi^{\prime}, \xi^{\prime}\right)-\beta y \frac{\partial u^{\prime}}{\partial z}=-\frac{\partial b^{\prime}}{\partial y}+D_{\xi^{\prime}}, \\
\frac{\partial b^{\prime}}{\partial t}+J\left(\psi^{\prime}, b^{\prime}\right)-N^{2} \frac{\partial \psi^{\prime}}{\partial y}=D_{b^{\prime}},
\end{gathered}
$$

where $J$ is the Jacobian operator $J(R, S)=(\partial R / \partial y)(\partial S / \partial z)-(\partial R / \partial z)(\partial S / \partial y)$ and terms $D$ correspond to dissipative/diffusive effects. The total fields are the zonal velocity $u=\gamma y \cos \left(\omega_{0} t\right)+u^{\prime}$, the meridional streamfunction $\psi=\psi^{\prime}$ and the rescaled density anomaly $b=b^{\prime}$.

\subsection{Linear parametric instability}

The non-dimensionalization of the equations of motion governing a given vertical mode $m, t=\sqrt{(m / N \beta)} t^{*}, y=\sqrt{(N / m \beta)} y^{*}, \gamma=\sqrt{(N \beta / m)} \gamma^{*}, \omega_{0}=\sqrt{(N \beta / m)} \omega_{0}^{*}$, (asterisks are dropped in the rest of this section), yields for the hydrostatic $\left(\xi^{\prime}=\nabla^{2} \psi^{\prime} \sim\right.$ $\left.\partial^{2} \psi^{\prime} / \partial z^{2}\right)$ inviscid $(D=0)$ linearized zonal vorticity equation

$$
\frac{\partial^{2} \psi^{\prime}}{\partial t^{2}}+y^{2} \psi^{\prime}-\frac{\partial^{2} \psi^{\prime}}{\partial y^{2}}-\gamma y \cos \left(\omega_{0} t\right) \psi^{\prime}=0
$$

The meridional streamfunction $\psi^{\prime}(y, t)$ is projected onto the basis $\varphi_{n}(y)=H_{n}(y)$ $\exp \left(-y^{2} / 2\right)$ where $H_{n}(y)$ is the $n$th Hermite polynomial

$$
\psi^{\prime}(y, t)=\sum_{n \geqslant 0} \varphi_{n}(y) \phi_{n}(t)
$$

Since the meridional modes verify $\partial^{2} \varphi_{n} / \partial y^{2}-y^{2} \varphi_{n}=-\delta_{n}^{2} \varphi_{n}$ with $\delta_{n}^{2}=2 n+1$, this leads to

$$
\sum_{n \geqslant 0}\left(\varphi_{n} \frac{\partial^{2} \phi_{n}}{\partial t^{2}}+\delta_{n}^{2} \varphi_{n} \phi_{n}-\gamma y \varphi_{n} \phi_{n} \cos \left(\omega_{0} t\right)\right)=0 .
$$


Making use of the Hermite polynomials recurrence relation $y H_{n}=n H_{n-1}+H_{n+1} / 2$ and of the Hermite functions orthogonality $\left(\int \varphi_{l}(y) \varphi_{n}(y) \mathrm{d} y=0\right.$ if $l \neq n$ and $2^{n} n ! \sqrt{\pi}$ if $l=n$ ), each meridional mode $n$ verifies

$$
\frac{\partial^{2} \phi_{n}}{\partial t^{2}}+\delta_{n}^{2} \phi_{n}=\gamma\left(\frac{1}{2} \phi_{n-1}+(n+1) \phi_{n+1}\right) \cos \left(\omega_{0} t\right),
$$

where $\phi_{-1}=0$ is assumed.

Denoting

$$
\begin{aligned}
\Phi(t) & =\left(\begin{array}{c}
\phi_{0}(t) \\
\phi_{1}(t) \\
. . \\
. . \\
\phi_{n}(t) \\
. .
\end{array}\right), \quad \Delta^{2}=\left[\begin{array}{cccccc}
\delta_{0}^{2} & 0 & & & & \\
0 & \delta_{1}^{2} & 0 & & & \\
& . . & . . & . . & & \\
& & . & . . & . . & \\
& & & 0 & \delta_{n}^{2} & 0 \\
& & & . . & . .
\end{array}\right], \\
H & =\left[\begin{array}{llllll}
0 & 1 & & & \\
\frac{1}{2} & 0 & 2 & & & \\
& . . & . . & . . & & \\
& . . & . . & . . & \\
& & \frac{1}{2} & 0 & n+1 \\
& & & . . & . .
\end{array}\right],
\end{aligned}
$$

the system (3.5) can be rewritten as an infinite dimensional system that only involves time-dependent coefficients

$$
\frac{\partial^{2}}{\partial t^{2}}(\Phi)+\left[\Delta^{2}-\gamma H \cos \left(\omega_{0} t\right)\right](\Phi)=0 .
$$

Except for the infinite dimension of the system, the structure of the above equation is similar to the Mathieu-type equation (2.5). We may thus anticipate that the excitation frequency $\omega_{0}$ will determine which meridional mode $n$ can resonate, and that the shear magnitude $\gamma$ will control the growth rate of the instability. This is precisely the purpose of the multiple-scale analysis of $\S 3.1 .1$.

\subsubsection{Multiple-scale analysis for $\gamma \ll 1$}

If $\gamma=0$, each meridional mode $n$ is independent of other modes

$$
\frac{\partial^{2} \phi_{n}}{\partial t^{2}}+\delta_{n}^{2} \phi_{n}=0
$$

Each free mode oscillates at frequency $\delta_{n}$. Such oscillations result from the combination of two restoring forces due to the stratification $N$ and to the Earth's rotation $f=\beta y$, while their equatorial trapping results from the $\beta$-effect.

Following Nayfeh \& Mook (1979, p. 307), we shall perform a multiple-scale analysis for a given oscillating shear frequency $\omega_{0}$ such that $\gamma \ll 1$.

The slow time variable is defined as $\tau=\gamma t$. For each meridional mode $n$

$$
\phi_{n}(t, \tau)=A_{n}^{0}(t, \tau)+\gamma A_{n}^{1}(t, \tau)+O\left(\gamma^{2}\right),
$$

$\left(A_{-1}^{0}=A_{-1}^{1}=0\right.$ is assumed $)$.

The zeroth-order equation is

$$
\frac{\partial^{2} A_{n}^{0}}{\partial t^{2}}+\delta_{n}^{2} A_{n}^{0}=0,
$$


thus

$$
A_{n}^{0}(t, \tau)=\mathrm{B}_{n}(\tau) \exp \left(\mathrm{i} \delta_{n} t\right)+\text { c.c. }
$$

$\left(\mathrm{B}_{-1}=0\right.$ is assumed and c.c. denotes the complex conjugate since $\mathrm{B}_{n}$ is complex).

The first-order equation is

$$
\frac{\partial^{2} A_{n}^{1}}{\partial t^{2}}+\delta_{n}^{2} A_{n}^{1}=-\left(2 \frac{\partial^{2} A_{n}^{0}}{\partial \tau \partial t}\right)+\left(\frac{1}{2} A_{n-1}^{0}+(n+1) A_{n+1}^{0}\right) \cos \left(\omega_{0} t\right),
$$

thus, replacing $A_{n}^{0}$ with its expression found at zeroth order,

$$
\begin{aligned}
\frac{\partial^{2} A_{n}^{1}}{\partial t^{2}}+\delta_{n}^{2} A_{n}^{1}= & -2 \mathrm{i} \delta_{n} \frac{\partial \mathrm{B}_{n}}{\partial \tau} \exp \left(\mathrm{i} \delta_{n} t\right) \\
& +\frac{1}{4} \mathrm{~B}_{n-1}(\tau)\left(\exp \left(\mathrm{i}\left(\delta_{n-1}+\omega_{0}\right) t\right)+\exp \left(\mathrm{i}\left(\delta_{n-1}-\omega_{0}\right) t\right)\right) \\
& +\frac{1}{2}(n+1) \mathrm{B}_{n+1}(\tau)\left(\exp \left(\mathrm{i}\left(\delta_{n+1}+\omega_{0}\right) t\right)+\exp \left(\mathrm{i}\left(\delta_{n+1}-\omega_{0}\right) t\right)\right)+\text { c.c. }
\end{aligned}
$$

Secular terms in $t$ in the order 1 equation correspond to the right-hand side forcing terms that are solutions of the homogenous equation and are proportional to $\exp \left(\mathrm{i} \delta_{n} t\right)$ or c.c. The cancellation of such secular terms yields the time-evolution equation for $\mathrm{B}_{n}$ as a function of $\tau$. We can distinguish several cases.

(i) Non-resonant meridional modes

For a meridional mode $n$ such that $\omega_{0}$ is far from $\delta_{n \pm 1} \pm \delta_{n}$, secular terms will cancel out if $\partial \mathrm{B}_{n} / \partial \tau=0$, thus

The solution $A_{n}^{1}$ is then

$$
\mathrm{B}_{n}(\tau)=\mathrm{B}_{n}
$$

$$
\begin{aligned}
A_{n}^{1}(t, \tau)= & -\frac{1}{4} \mathrm{~B}_{n-1}\left(\frac{\exp \left(\mathrm{i}\left(\delta_{n-1}+\omega_{0}\right) t\right)}{\left(\delta_{n-1}+\omega_{0}\right)^{2}-\delta_{n}^{2}}+\frac{\exp \left(\mathrm{i}\left(\delta_{n-1}-\omega_{0}\right) t\right)}{\left(\delta_{n-1}-\omega_{0}\right)^{2}-\delta_{n}^{2}}\right) \\
& -\frac{1}{2}(n+1) \mathrm{B}_{n+1}\left(\frac{\exp \left(\mathrm{i}\left(\delta_{n+1}+\omega_{0}\right) t\right)}{\left(\delta_{n+1}+\omega_{0}\right)^{2}-\delta_{n}^{2}}+\frac{\exp \left(\mathrm{i}\left(\delta_{n+1}-\omega_{0}\right) t\right)}{\left(\delta_{n+1}-\omega_{0}\right)^{2}-\delta_{n}^{2}}\right)+\text { c.c. }
\end{aligned}
$$

The order 1 response will be independent of $\tau$.

(ii) Possible resonant meridional modes

For a mode $n$ such that $\omega_{0}$ is close to $\omega_{r}=\delta_{n \pm 1} \pm \delta_{n}$ other terms become important and can lead to two cases of resonance. Introducing the detuning $b \gamma$ of the forcing frequency as

$$
\omega_{0}=\omega_{r}+\mathrm{b} \gamma+O\left(\gamma^{2}\right) .
$$

(a) $\omega_{r}=\delta_{n+1}+\delta_{n}$. Combination of the summed type.

Denoting with ${ }^{\dagger}$ a complex conjugate, differential equations for the slow time evolution of modes $n$ and $n+1$ are

$$
\begin{gathered}
\frac{\partial \mathrm{B}_{n}}{\partial \tau}=-\mathrm{i} \frac{1}{2 \delta_{n}} \frac{n+1}{2} \mathrm{~B}_{n+1}^{\dagger}(\tau) \mathrm{e}^{\mathrm{i} b \tau}, \\
\frac{\partial \mathrm{B}_{n+1}}{\partial \tau}=-\mathrm{i} \frac{1}{2 \delta_{n+1}} \frac{1}{4} \mathrm{~B}_{n}^{\dagger}(\tau) \mathrm{e}^{\mathrm{ib} \tau} .
\end{gathered}
$$

Non-trivial solutions of the system are

$$
\begin{gathered}
\mathrm{B}_{n}(\tau)=a_{n} \mathrm{e}^{-\mathrm{i} \lambda \tau}, \\
\mathrm{B}_{n+1}(\tau)=a_{n+1} \mathrm{e}^{\mathrm{i}\left(\lambda^{\dagger}+\mathrm{b}\right) \tau},
\end{gathered}
$$


with

$$
\lambda=-\frac{1}{2}\left[\mathrm{~b} \pm\left(\mathrm{b}^{2}-\frac{n+1}{8} \frac{1}{\delta_{n} \delta_{n+1}}\right)^{1 / 2}\right] .
$$

Solutions remain bounded if and only if

$$
\mathrm{b}^{2}>\frac{n+1}{8} \frac{1}{\delta_{n} \delta_{n+1}} .
$$

For $\mathrm{b}^{2}=(n+1) / 8 \delta_{n} \delta_{n+1}$, the solution has an algebraic growth behaviour like $\tau \mathrm{e}^{-\mathrm{ib} \tau / 2}$. In the $\left(\omega_{0}, \gamma\right)$-plane, the marginal curve separating stable and unstable cases is

$$
\omega_{0}=\delta_{n}+\delta_{n+1} \pm \gamma\left(\frac{n+1}{8} \frac{1}{\delta_{n} \delta_{n+1}}\right)^{1 / 2}+O\left(\gamma^{2}\right) .
$$

For $\mathrm{b}^{2}<(n+1) / 8 \delta_{n} \delta_{n+1}$, mode $n$ has an exponential growth rate

$$
\mu=\frac{1}{2} \sqrt{\gamma^{2} \frac{n+1}{8} \frac{1}{\delta_{n} \delta_{n+1}}-\left(\omega_{0}-\delta_{n}-\delta_{n+1}\right)^{2}}
$$

(where $\omega_{0}=\delta_{n+1}+\delta_{n}+\mathrm{b} \gamma$ has been used).

(b) $\omega_{r}=\delta_{n+1}-\delta_{n}$. Combination of the difference type.

A similar analysis to the previous case shows that there can be no resonance.

At leading order in $\gamma$, the only possible resonances correspond to $\omega_{r}=\delta_{n}+\delta_{n+1}$, a combination resonance of the summed type. They correspond to a subharmonic resonance similar to the classical Mathieu equation, since a frequency $\omega_{0}$ is able to excite a combination of modes with lower frequencies $\delta_{n}$ and $\delta_{n+1}<\omega_{0}$. Figure 2 provides the non-dimensional growth rate as a function of $\gamma$ and $\omega_{0}$ for a given vertical mode $m$.

Thus for a given vertical mode, we have recovered the properties of Mathieu's equation: (i) the excitation frequency $\omega_{0}$ determines which meridional mode $n$ can resonate, and (ii) the shear magnitude $\gamma$ controls the instability growth rate, but not which mode is excited.

The dimensional growth rate of an unstable vertical mode $m=\mathrm{p} N \beta / \omega_{0}^{2}$ and the corresponding meridional mode $n$ is

$$
\mu=\frac{1}{2} \gamma \sqrt{\frac{(n+1)}{8 \sqrt{(2 n+1)(2 n+3)}}-\frac{\omega_{0}^{2}}{\gamma^{2}}\left(1-\sqrt{\frac{2 n+1}{\mathrm{p}}}-\sqrt{\frac{2 n+3}{\mathrm{p}}}\right)^{2}}
$$

(where $\mathrm{p}$ is a positive real number and in the limit $\gamma \ll \omega_{0} / \sqrt{\mathrm{p}}$ ).

Note that higher-order effects in $\gamma^{q}(q>1)$ can be evaluated for improving the accuracy of the transition curve and also for assessing other resonances of order $q$; superharmonic resonances as in Mathieu's equation such that $q \omega_{r}=\delta_{n}+\delta_{n+1}$ will also appear at order $q$, but with much weaker growth rates as in the classical Mathieu's case (for $q=2$ terms see Nayfeh \& Mook 1979, p. 310).

\section{Vertical viscosity/diffusion effect}

Taking into account vertical viscosity amounts to replace $\partial / \partial t$ by $\partial / \partial t+v\left(\partial^{2} / \partial z^{2}\right)$ dimensionally. For a given vertical mode $m$, the non-dimensional equation becomes

$$
\frac{\partial^{2} \psi^{\prime}}{\partial t^{2}}+2 \varepsilon \frac{\partial \psi^{\prime}}{\partial t}+\varepsilon^{2} \psi^{\prime}+y^{2} \psi^{\prime}-\frac{\partial^{2} \psi^{\prime}}{\partial y^{2}}-\gamma y \cos \left(\omega_{0} t\right) \psi^{\prime}=0
$$



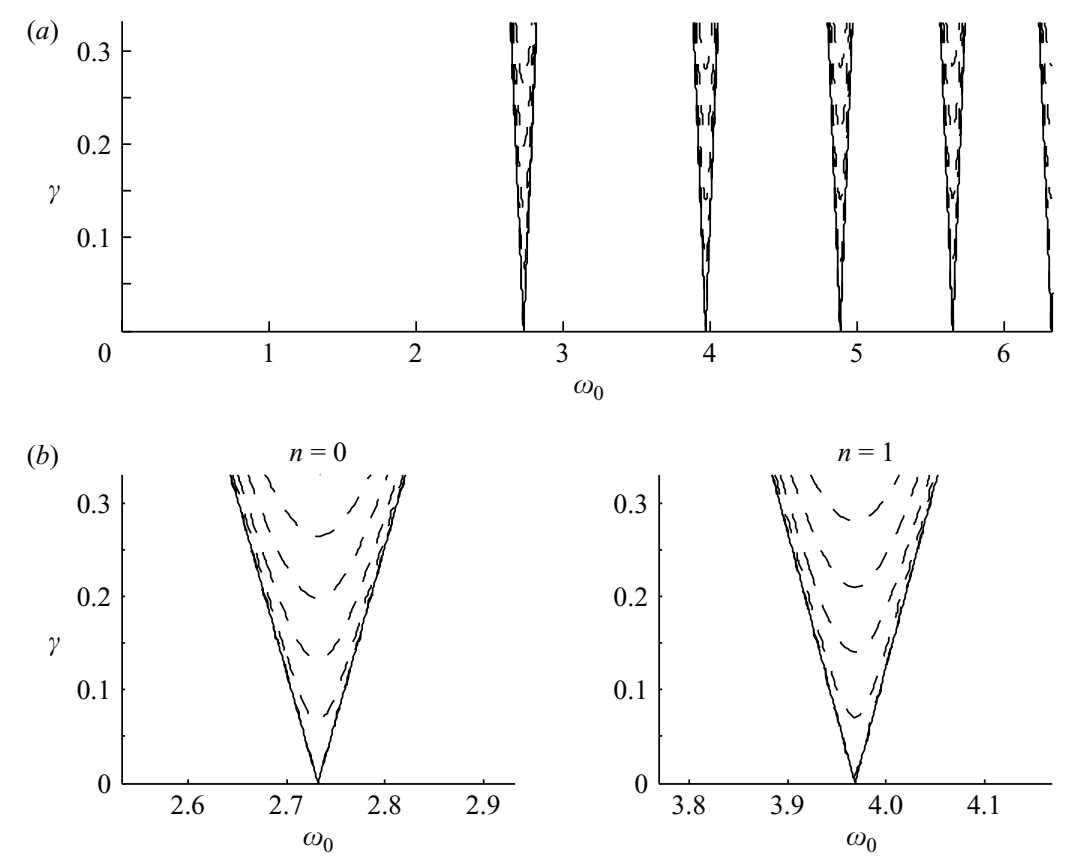

FIGURE 2. Growth rate $\mu$ for an oscillating shear. All quantities are non-dimensionalized assuming a given vertical mode $m$. Solid line : transition curves. Dashed lines : contour interval every 0.01. (a) Visualization in the global $\left(\omega_{0}, \gamma\right)$-plane. (b) Zoom of the unstable zone corresponding to $n=0$ and $n=1$ showing the comparable growth rates for each resonance band for a given vertical mode.

with $\varepsilon=\sqrt{\nu^{2} m^{5} / N_{0} \beta}$. Each meridional mode satisfies

$$
\frac{\partial^{2} \phi_{n}}{\partial t^{2}}+2 \varepsilon \frac{\partial \phi_{n}}{\partial t}+\left(\delta_{n}^{2}+\varepsilon^{2}\right) \phi_{n}=\gamma\left(\frac{1}{2} \phi_{n-1}+(n+1) \phi_{n+1}\right) \cos \left(\omega_{0} t\right)
$$

Introducing the new function $\chi_{n}(t)=\phi_{n}(t) \exp (-\varepsilon t)$, we obtain the same equation as in the previous case,

$$
\frac{\partial^{2} \chi_{n}}{\partial t^{2}}+\delta_{n}^{2} \chi_{n}=\gamma\left(\frac{1}{2} \chi_{n-1}+(n+1) \chi_{n+1}\right) \cos \left(\omega_{0} t\right) .
$$

Previous inviscid resonance results will hold, provided $\mu>\varepsilon$, or $\mu>v_{z} m^{2}$ dimensionally, which means that for a given shear strength $\gamma$, vertical viscosity will inhibit the parametric resonance of vertical modes that are too high.

\section{Physical interpretation}

In the absence of forcing, the solutions of the zonally symmetric system are the mixed Rossby-gravity and inertio-gravity waves. The existence of such free oscillating modes is due to the combined action of two restoring forces, stratification $N$ and Earth's rotation $f=\beta y$. It has been shown in $\S 2$ that a barotropic zonal shear oscillating at frequency $\omega_{0}$ brings about the possibility of a parametric instability that involves some of the free modes. The free-mode selection will be performed by the forcing frequency $\omega_{0}$.

In the case of small amplitude shear $\gamma$, the free modes that can be perfectly resonant are countably infinite and correspond to vertical modes $m$ for which there exist an $n$ 

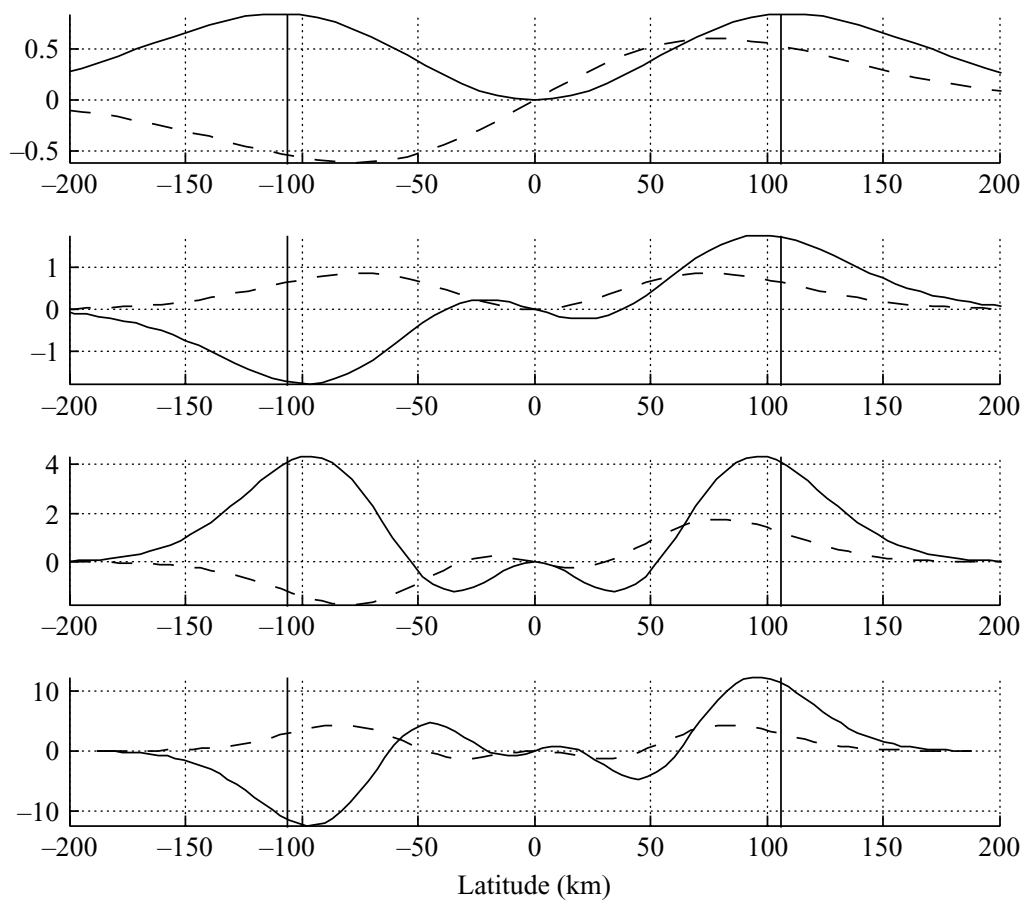

FIGURE 3. Meridional structure of the perturbation zonal velocity $u^{\prime}$ for perfectly resonating vertical modes such that $2 \pi / \omega_{0}=15$ days $\left(N=2 \times 10^{-3} \mathrm{~s}^{-1}\right.$ and $\left.\beta=2.29 \times 10^{-11} \mathrm{~m}^{-1} \mathrm{~s}^{-1}\right)$ in the stratified case. Top to bottom $n=0,1,2,3$. Associated vertical wavelengths are, respectively, $h=433 \mathrm{~m}, 205 \mathrm{~m}, 136 \mathrm{~m}, 111 \mathrm{~m}$. Continuous lines correspond to meridional mode $n+1$ and dotted lines to meridional mode $n$. Latitudes such that $\beta y_{c}= \pm \omega_{0} / 2$ are indicated by full vertical lines. Amplitudes are arbitrary.

such that the detuning is exactly zero

$$
\omega_{0}=(\sqrt{2 n+1}+\sqrt{2 n+3}) \sqrt{\frac{\beta N}{m}} .
$$

Their resonance with the basic state will involve the combination resonance of the summed type of two free meridional modes $n$ and $n+1$ for a given vertical mode $m$.

This mode selection is linked to the latitude where the inertial frequency $\beta y$ is half the forcing frequency $y_{c}=\omega_{0} / 2 \beta$; figure 3 presents the meridional structure of zonal velocity for the four lowest vertical modes that will resonate for a fixed oscillating shear frequency $\omega_{0}$. It is obvious in this figure that extrema of zonal velocity occur near the latitudes $y_{c}= \pm \omega_{0} / 2 \beta$, which are marked by a vertical line. As already seen in $\S 2$, such latitudes are those where parametric instability occurs in the barotropic case. We are therefore led to the inference that the 'driving' of the instability in this stratified case is actually an inertial resonance. The main role of stratification is to determine how the baroclinic meridional pressure gradient can adjust itself to such an inertial resonance: its adjustment leads to a vertical mode selection (3.7).

The 'driving' of the instability by a barotropic oscillating shear modifies the effective Coriolis force (term $\left(\beta y-\gamma \cos \left(\omega_{0} t\right)\right) v$ in (3.1)) that acts on the perturbation. Since this effective Coriolis force is the restoring force that appears in Mathieu's equation, the parametric instability which is studied here corresponds to a resonance of free 
inertial oscillations, rather than a resonance of free internal waves as in Majda \& Shefter $(1998 a, b)$.

We propose to name such resonant instability an equatorial inertial-parametric instability because (i) of its overall similarity with the classical steady inertial instability triggered by non-zero shear at the equator, and (ii) it corresponds to the resonant excitation of free inertio-gravity waves of the equatorial $\beta$-plane.

\subsection{Parametric instability $\left(\gamma \ll \omega_{0}\right)$ vs. inertial instability limit $\left(\gamma \gg \omega_{0}\right)$}

For large shear values such that $\gamma \gg \omega_{0}$, we recover the equatorial inertial instability limit since the oscillating shear period is much longer than the inertial instability growth rate and the latter will be able to develop fully before the shear reverses sign. Hereinafter we briefly summarize the main results of linear inertial instability and contrast them with those of linear parametric instability documented in the previous subsection.

\section{(a) Inertial instability $\left(\gamma \gg \omega_{0}\right)$}

The growth rate of linear inviscid inertial instability is a function of the vertical mode $m$ and of meridional mode $n$ (Dunkerton 1981; Stevens 1983)

$$
\mu(m, n)=\sqrt{\frac{\gamma^{2}}{4}-(2 n+1) \frac{N \beta}{m}} .
$$

If $m>4 N \beta / \gamma^{2}$, the growth rate $\mu(m, 0)$ is the largest for the smallest vertical scales $(m \rightarrow \infty)$ and tends toward

$$
\mu_{\max }^{I I}=\frac{1}{2} \gamma .
$$

Linear inertial instability thus has an ultraviolet character, with an excitation of a continuous spectrum of vertical modes ranging from infinity to the lowest inertially unstable mode

$$
m_{b}=\frac{4 N \beta}{\gamma^{2}} .
$$

The range of inertially unstable vertical modes is continuous (dashed line in figure $4 a$ ) with no clear vertical scale selection. This range directly depends on the shear strength $\gamma$, as seen in (3.8).

In the presence of vertical viscosity and diffusion, Dunkerton (1981) shows that there exists a critical shear value $\gamma_{c}$ for linear inertial instability

$$
\gamma_{c}^{I I}=\sqrt{5}\left(2 v N^{2} \beta^{2}\right)^{1 / 5},
$$

associated with a critical vertical mode

$$
m_{c}^{I I}=\left(\frac{N \beta}{4 v^{2}}\right)^{1 / 5} .
$$

Both $\gamma_{c}^{I I}$ and $m_{c}^{I I}$ only depend on external parameters $(\beta, N)$ and on viscosity $v$. Viscosity thus introduces a vertical mode selection for inertial instability (solid line in figure $4 a$ ). See Griffiths (2003b) for further discussion on viscous vertical scale selection.

(b) Parametric instability $\left(\gamma \ll \omega_{0}\right)$

In the linear inviscid parametric instability case, a local maximal growth rate appears for each mode $(n, m)$ that satisfies the exact resonant condition (3.7), and the 

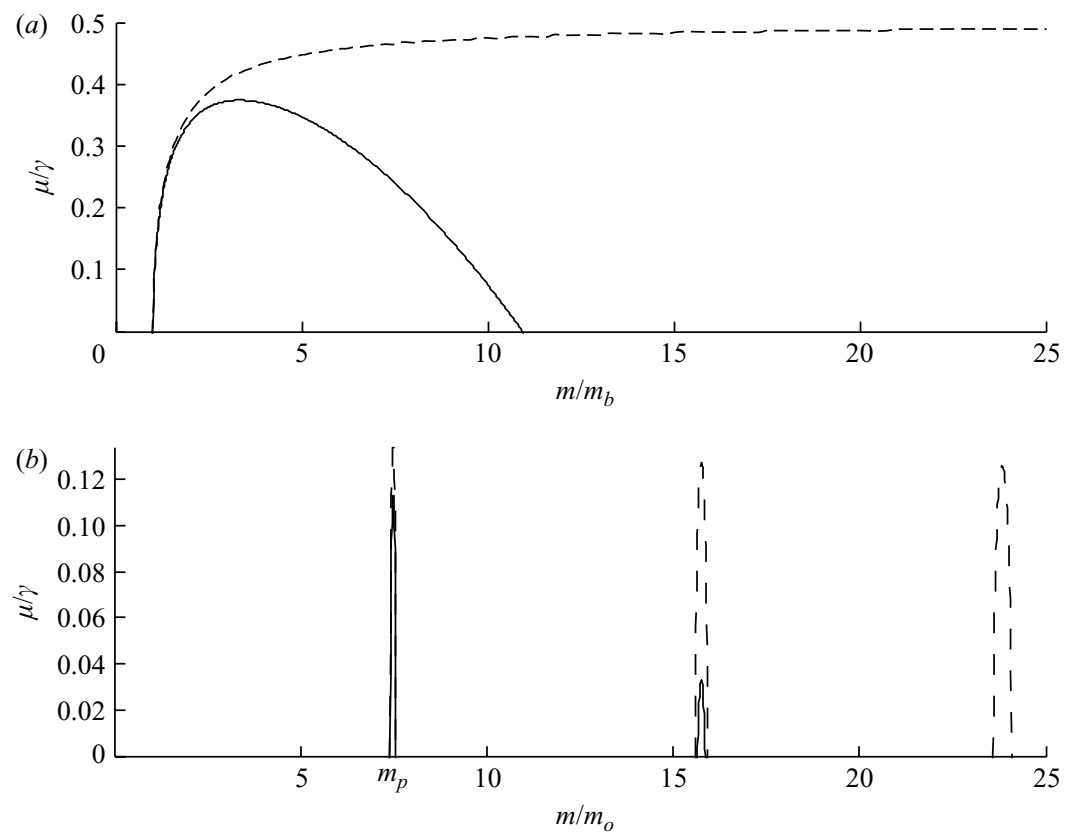

FiguRE 4. Growth rate as a function of vertical mode for both inertial and parametric instability. Parameters are $N=2 \times 10^{-3} \mathrm{~s}^{-1}, v_{z}=10^{-5} \mathrm{~m}^{2} \mathrm{~s}^{-1}$. (a) Inertial instability case: $\gamma=$ $2.4 \times 10^{-6} \mathrm{~s}^{-1}, m$ non-dimensionalized by $m_{b}=4 N \beta / \gamma^{2}$. (b) Parametric instability case: $\omega_{0}=4.8 \times 10^{-6} \mathrm{~s}^{-1}$ and $\gamma=1 \times 10^{-7} \mathrm{~s}^{-1}, \quad m$ is non-dimensionalized by $m_{0}=N \beta / \omega_{0}^{2}$ (characteristic vertical mode corresponding to the forcing frequency). Note that $\gamma$ and the non-dimensionalization differ between the two instabilities. ---, inviscid case; - , viscous case.

global maximum growth rate is

$$
\mu_{\max }^{P I}=\frac{\gamma}{4 \sqrt{2 \sqrt{3}}} \sim \frac{1}{8} \gamma
$$

corresponding to the lowest vertical unstable mode with $n=0$,

$$
m_{p}=(1+\sqrt{3})^{2} \frac{\beta N}{\omega_{0}^{2}} .
$$

Linear inviscid parametric instability thus leads to a discrete vertical mode spectrum (dashed line in figure $4 b$ ) and the inviscid mode selection is only a function of the forcing frequency $\omega_{0}$ and is independent of the shear amplitude $\gamma$.

In the presence of vertical viscosity and diffusion, we can introduce an approximate critical shear for parametric instability based on the destabilization of the lowest vertical $m_{p}$ mode $\left(\mu\left(m_{p}\right)>v m_{p}^{2}\right)$ so that

$$
\gamma_{c}^{P I} \sim 8 v \beta^{2} N^{2} \frac{(1+\sqrt{3})^{4}}{\omega_{0}^{4}} .
$$

In contrast to the inertial instability case for which $\gamma_{c}^{I I}$ depends only on external parameters $(\beta, N, v), \gamma_{c}^{P I}$ also depends on the dynamical forcing through $\omega_{0}^{4}$. (The faster the pulsation forcing is, the lower is the critical shear.) Moreover,

$$
m_{c}^{P I}=m_{p}=(1+\sqrt{3})^{2} m_{0} \approx 7.45 m_{0}, \quad m_{0}=\frac{\beta N}{\omega_{0}^{2}},
$$


is independent of viscosity $v$ (in contrast to $m_{c}^{I I}$ ), and is solely related to $m_{0}$, the equatorial vertical scale characteristic of frequency $\omega_{0}$. This unstable lowest vertical mode is clearly favoured by dissipation (solid line in figure $4 b$ ).

Although linear parametric instability provides a smaller growth rate than linear inertial instability, its properties are more physically relevant: (i) there is a clear linear inviscid vertical mode selection with a discrete spectrum which solely depends on the forcing pulsation $\omega_{0}$; (ii) moreover, vertical viscosity enhances this vertical scale selection towards the lowest vertical unstable mode; (iii) finally, in the viscous case, the critical shear is reduced when the forcing frequency is faster.

\section{Parametric destabilization of zonally symmetric mixed Rossby-gravity wave}

The previous section has provided evidence that a barotropic oscillating zonal shear flow could excite parametric resonances of high vertical free modes of the equatorial $\beta$-plane. For atmospheric and oceanic conditions, such an oscillating shear flow could be ascribed to large vertical-scale equatorial waves. For zonally symmetric conditions, the simplest wave presenting such oscillating shear is the mixed Rossbygravity (MRG) wave, which corresponds to an oscillating shear flow which is also of alternating sign in the vertical direction. We can wonder if such waves can also induce parametric excitation of higher vertical modes.

We shall investigate the case of a basic state corresponding to a standing MRG wave. The MRG wave frequency is $\omega_{0}$, its vertical wavenumber is $m_{0}$ and we define

$$
\begin{aligned}
c_{0} & =\frac{N}{m_{0}}, \\
\lambda_{0} & =\sqrt{\frac{N}{\beta m_{0}}}, \\
(\widetilde{V}, \widetilde{W}) & =\left(-\frac{\partial \widetilde{\Psi}}{\partial z}, \frac{\partial \widetilde{\Psi}}{\partial y}\right) .
\end{aligned}
$$

For a standing MRG wave,

$$
\begin{aligned}
(\widetilde{U}, \widetilde{B}, \widetilde{\Psi})= & V_{0} \exp \left(-\left(y^{2} / 2 \lambda_{0}^{2}\right)\right) \\
& \times\left(\frac{y}{\lambda_{0}} \cos \left(\omega_{0} t\right) \cos \left(m_{0} z\right), N \frac{y}{\lambda_{0}} \cos \left(\omega_{0} t\right) \sin \left(m_{0} z\right), \frac{1}{m_{0}} \sin \left(\omega_{0} t\right) \sin \left(m_{0} z\right)\right) .
\end{aligned}
$$

\subsection{Nonlinear initial-value simulation}

Such an MRG wave is prescribed with small random perturbations at $t=0$ as the initial condition of the fully nonlinear system integrated with the numerical model of Hua et al. (1997)

$$
\begin{gathered}
\frac{\partial u}{\partial t}+J(\psi, u)+\beta y \frac{\partial \psi}{\partial z}=D_{u}, \\
\frac{\partial \xi}{\partial t}+J(\psi, \xi)-\beta y \frac{\partial u}{\partial z}=-\frac{\partial b}{\partial y}+D_{\xi}, \\
\frac{\partial b}{\partial t}+J(\psi, b)-N^{2} \frac{\partial \psi}{\partial y}=D_{b},
\end{gathered}
$$

Note that since $(u, b, \psi)$ denotes the total fields, they include the initialized MRG wave signal. 
The domain of integration spans $1000 \mathrm{~km}$ on each side of the equator and the domain height corresponds to a vertical wavelength of the MRG wave $H=2 \pi / m_{0}=1600 \mathrm{~m}$. Spatial scales $L_{y}=O(100 \mathrm{~km})$ and $H_{z}=O(100 \mathrm{~m})$, require a very anisotropic grid and therefore a hydrostatic assumption. The grid size is $d y=6 \mathrm{~km}$ and $d z=12.5 \mathrm{~m}$. Periodic conditions in the vertical are assumed. The constant stratification background is $N=3 \times 10^{-3} \mathrm{~s}^{-1}$ and the wave frequency is taken as $\omega_{0}=4.17 \times 10^{-6} \mathrm{~s}^{-1}$ (its period $T \approx 15$ days is typical of deep oceanic $M R G$ wave observations, e.g. Bunge et al. 2005). The radius of deformation of the basic MRG wave is $\lambda_{0}=1.8 \times 10^{5} \mathrm{~m}$ and its celerity is $c_{0}=0.76 \mathrm{~m} \mathrm{~s}^{-1}$.

Only very small dissipation and diffusion, necessary for the numerical stability of the model, are acting $\left(D=v_{z}\left(\partial^{2} / \partial z^{2}\right)+v_{4 h}\left(\partial^{4} / \partial y^{4}\right)\right.$ with $v_{z}=10^{-6} \mathrm{~m}^{2} \mathrm{~s}^{-1}$ and $v_{4 h}=$ $1 \times 10^{8} \mathrm{~m}^{4} \mathrm{~s}^{-1}$ ), and we recall that no external forcing is applied. Such initial-value simulation is therefore nearly conserving the invariants of the system such as angular momentum.

Denoting $\gamma$ as the maximum shear value of the MRG wave, the Froude number of the basic-state wave is

$$
\alpha=\frac{\gamma}{\omega_{0}}=\frac{V_{0}}{c_{0}} .
$$

In this section and the following one, results are presented for a MRG wave of amplitude $V_{0}=0.15 \mathrm{~m} \mathrm{~s}^{-1}$, thus implying a Froude number of $\alpha \sim 0.2$. We have furthermore checked that the results which are reported next are also valid for $\alpha$ ranging from 0.03 to 0.4 .

\section{Transient adjustment phase}

It is well known that linear waves of the equatorial $\beta$-plane are not exact solutions of the nonlinear equations of motion, in contrast to the linear quasi-geostrophic waves of the mid-latitude $\beta$-plane or with linear internal waves. As expected, the numerical simulations show in a first phase that the flow adjusts itself by emitting gravity waves with a broad vertical mode spectrum. Low vertical modes propagate far away from the equator whereas high vertical modes remain trapped between the turning latitudes $y_{t}= \pm \omega_{0} / \beta$ (Anderson \& Gill 1979). These equatorially-trapped waves, which are observed to have high meridional wavenumbers, are compatible with the WKB analysis of Gill (1982, p. 441). They have frequencies $\omega=p \omega_{0}$ with integer $p \geqslant 1$, and are thus not subharmonic resonantly excited. Although their amplitude is found to grow initially, they saturate nonlinearly to weak amplitude after $O(1500$ days). Although the saturation amplitude is found to be a growing function of the Froude number $\alpha$, this first adjustment phase has little impact on the final adjustment phase, for the entire Froude number range that has been explored.

\section{Final adjustment phase}

After the observed saturation of the trapped gravity waves, high vertical inertiogravity modes are excited with a slow growth rate which is, however, sustained so that they eventually dominate the baroclinic response which is superposed on the adjusted basic state $\mathrm{MRG}$ wave. In contrast to the trapped gravity waves, the observed inertio-gravity waves have low meridional wavenumbers.

Figure 5 displays the zonal velocity and density perturbation fields of such modes for their nonlinear saturation after filtering out the $m_{0}$ mode signal with the same vertical wavelength as the basic state $\mathrm{MRG}$ wave. Extrema of zonal velocity perturbations are located near the critical inertial latitudes $\left(y_{c}= \pm \omega_{0} / 2 \beta\right)$. The filtered flow is dominated by vertical modes $m / m_{0}=7$ and 8 , as corroborated by the clear peak 


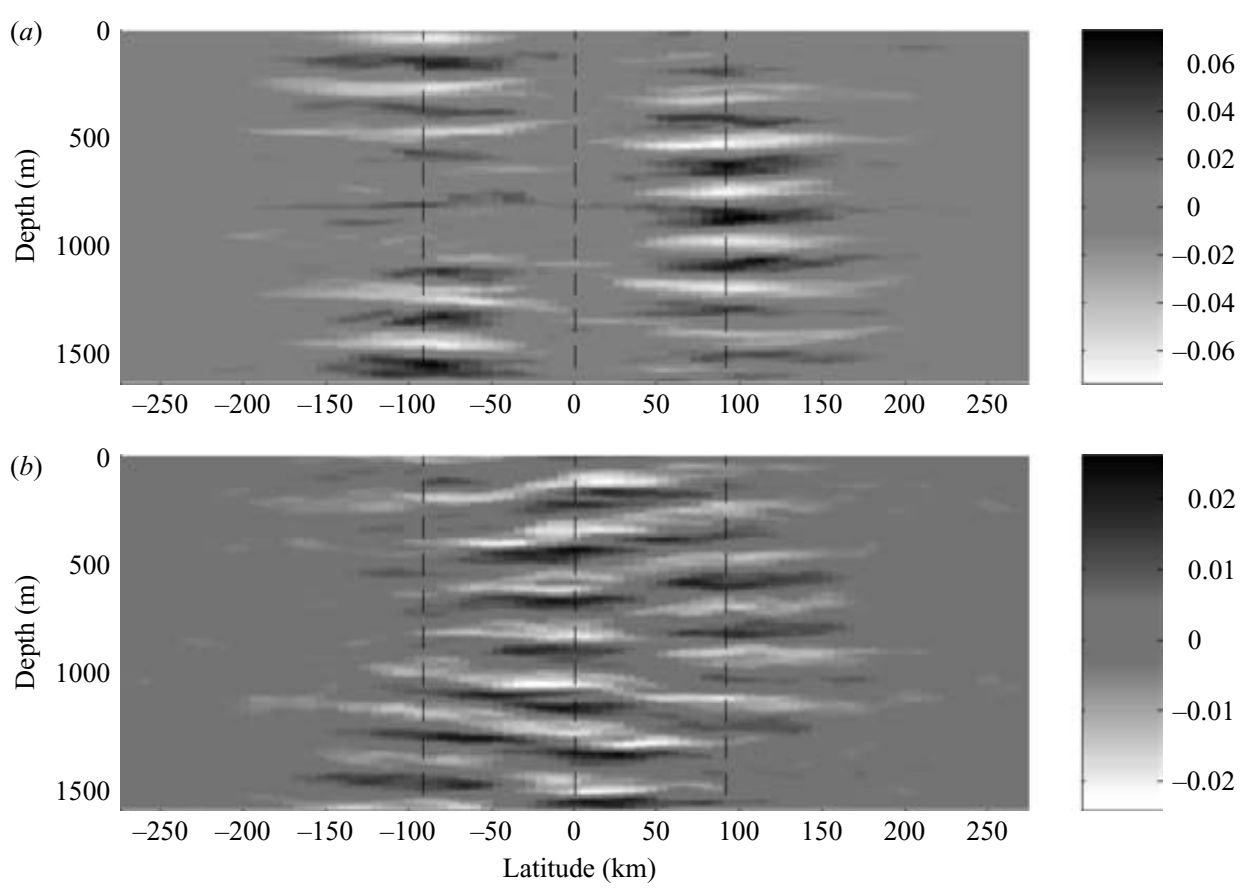

Figure 5. Final adjustment phase of an MRG wave destabilization: (a) zonal velocity and (b) density anomaly fields for which the signal at the vertical $m_{0}$ wavenumber has been filtered out. Dashed lines indicate the equator and the critical inertial latitudes $y_{c}= \pm \omega_{0} / 2 \beta$.

(a)

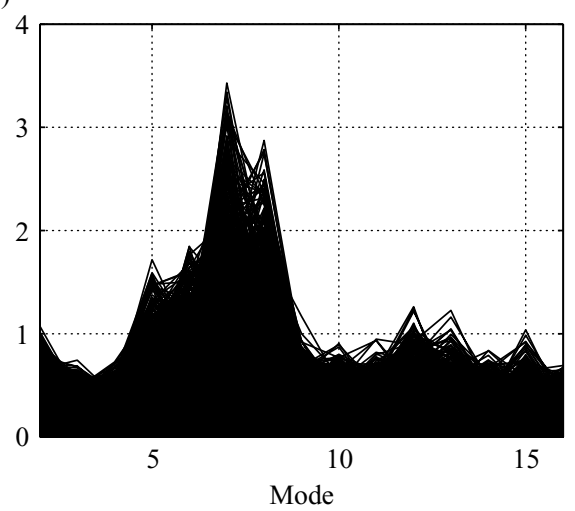

(b)

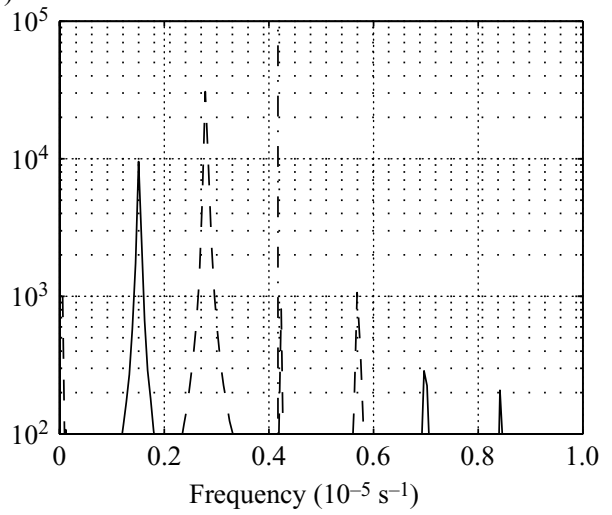

FIGURE 6. Spectra of $u$ at the critical inertial latitude $y_{c}= \pm \omega_{0} / 2 \beta$ for the MRG wave initial-value simulation. (a) Vertical mode spectra at different instants of the simulation. (b) Frequency spectra of vertical modes 7 (dashed line) and 8 (solid line) during the final adjustment phase. The vertical dash-dotted line indicates the frequency value $\omega_{0}$ of the basic state MRG wave.

displayed by the vertical mode spectra at different instants of the simulation shown in figure $6(a)$. Moreover, a projection of the signal onto Hermite functions reveals that the vertical mode $m / m_{0}=7$ corresponds to a meridional wavenumber $n=1$, while vertical mode $m / m_{0}=8$ has a meridional wavenumber $n=0$. Since a structure with vertical wavenumber $m$ and meridional mode $n$ has frequency $\omega_{n, m}=\sqrt{(2 n+1) N \beta / m}$, 
(a)

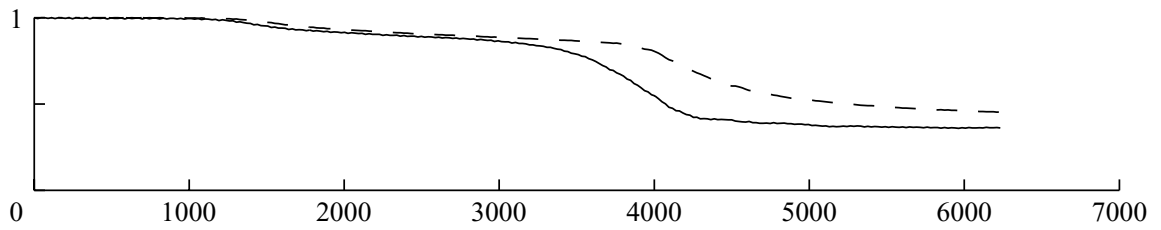

(b)

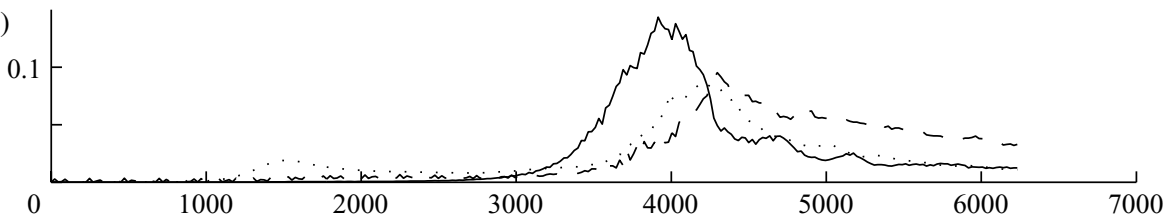

(c)

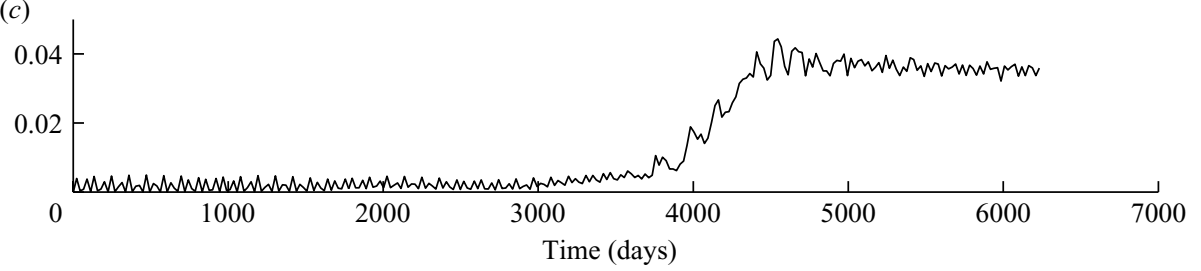

FiguRE 7. MRG wave initial-value simulation. Plots of total energy versus time scaled by the initial energy. (a) Energy of MRG vertical mode $m_{0}$ (solid line) and energy of all modes (dashed line). (b) Energy of the pair $m=7 m_{0}$ and $m=8 m_{0}$ (solid line), of modes $m>8 m_{0}$ (dotted line) and of modes $m_{0}<m<7 m_{0}$ (dashed line). (c) Energy of the barotropic mode.

a summed resonance of the structures $n=1, m=7 m_{0}$ and $n=0, m=8 m_{0}$ is possible

$$
\omega_{\left\{1,7 m_{0}\right\}}+\omega_{\left\{0,8 m_{0}\right\}} \approx \omega_{0},
$$

involving different meridional wavenumbers of two distinct vertical modes. The frequency spectra of both vertical modes shown in figure $6(b)$ confirms the possibility of a parametric combination resonance of the summed type.

\section{Energy exchange}

In order to study the energy exchanges that take place during the initial-value simulation, plots of energy as a function of time and for various vertical modes are provided in figure 7 . Note that figure 5 is plotted at 3500 days at which modes $m / m_{0}=7$ and 8 have undergone a significant growth (figure $7 b$ ).

As seen in figure 7(a), up to 3000 days the total amount of energy (dashed line) corresponds almost entirely to the energy of the vertical mode $m_{0}$ of the MRG wave (solid line). The transient adjustment phase has therefore weakly perturbed the flow. Between 3000 and 4000 days, the sustained growth of vertical modes $m / m_{0}=7$ and 8 is obvious (figure $7 b$, solid line), and extracts its energy from the $m_{0}$ energy which deviates from the total amount of energy.

Near 4000 days, modes $m / m_{0}=7$ and 8 break and this has two consequences: (i) a strong dissipation of the total amount of energy (figure $7 a$, dashed line) and (ii) an irreversible growth of the barotropic mode (figure $7 c$ ).

Pursuing the integration further in time, a slow dissipative/diffusive decay of all the signals is observed, but note that vertical modes $m_{0}, 7 m_{0}, 8 m_{0}$ and the barotropic mode predominate. 
We shall next provide a rationale for the growth of modes $m / m_{0}=7$ and 8 in terms of a subharmonic inertial-parametric instability. The consequences of the breaking of these modes in terms of redistribution of angular momentum are discussed in $\S 5$.

\subsection{Linear parametric destabilization of the mixed Rossby-gravity wave}

In order to demonstrate the inertial-parametric character of the MRG wave destabilization, the equations of motion are linearized around the basic-state wave. To make notations clear in this section, 4.2, and in Appendix B.1, we shall denote partial derivatives with a subscript. The linearized system can be written as

$$
\begin{gathered}
u_{t}^{\prime}+\beta y \psi_{z}^{\prime}=-J\left(\widetilde{\Psi}, u^{\prime}\right)-J\left(\psi^{\prime}, \widetilde{U}\right), \\
b_{t}^{\prime}-N^{2} \psi_{y}^{\prime}=-J\left(\widetilde{\Psi}, b^{\prime}\right)-J\left(\psi^{\prime}, \widetilde{B}\right), \\
\psi_{z z t}^{\prime}-\beta y u_{z}^{\prime}+b_{y}^{\prime}=-J\left(\widetilde{\Psi}, \psi_{z z}^{\prime}\right)-J\left(\psi^{\prime}, \widetilde{\Psi}_{z z}\right),
\end{gathered}
$$

where self-advection terms of the MRG wave are discarded, since they correspond to vertical modes $m=2 m_{0}$ while our focus is on the excitation of high vertical modes.

A direct numerical integration of system (4.6)-(4.8), with small initial random perturbations, shows the growth of gravity waves with high vertical modes that are trapped between the turning latitudes, as seen in the first adjustment phase of the nonlinear initial-value simulation of $\S 4.1$, where they quickly saturate with weak amplitudes and do not dominate the final state. Since (4.6)-(4.8) are linear, such excited gravity waves cannot saturate nonlinearly in the present case. A reduction of the above system is thus required in order to identify possible subharmonic parametric resonances which dominate the final phase of the nonlinear initial-value simulation.

Since figure 7 suggests important energy exchanges between the MRG wave and higher vertical modes, let us examine the energy gain by the perturbations in the above linearized system. Denoting

$$
K^{\prime}=\frac{u^{\prime 2}+v^{\prime 2}}{2}, \quad P^{\prime}=\frac{b^{\prime 2}}{2 N^{2}}, \quad E^{\prime}=K^{\prime}+P^{\prime}
$$

as, respectively, the kinetic, potential and total energy of the perturbation, the domainaveraged energy budget, for vertically periodic conditions and perturbations going to 0 as $y$ tends to infinity, is

$$
\frac{\partial}{\partial t} \iint E \mathrm{~d} y \mathrm{~d} z=-\iint\left[u^{\prime} J\left(\psi^{\prime}, \widetilde{U}\right)+v^{\prime} J\left(\psi^{\prime}, \widetilde{V}\right)+\frac{b^{\prime}}{N^{2}} J\left(\psi^{\prime}, \widetilde{B}\right)\right] \mathrm{d} y \mathrm{~d} z .
$$

The above equation reveals that the energy exchange terms are solely due to the second terms in the right-hand side of system (4.6)-(4.8). They correspond to the advection by the perturbation of the MRG wave's properties. The first terms in the right-hand side of system (4.6)-(4.8) (advection by the MRG wave of the perturbation) only redistribute spatially the perturbation energy, but do not contribute to its growth on spatial average, albeit they are much larger in amplitude than the second terms if a scaling were performed.

The relevant truncated linearized system of equations for perturbations which can therefore be parametrically excited by the basic-state MRG wave is thus

$$
\begin{gathered}
u_{t}^{\prime}+\beta y \psi_{z}^{\prime}=-J\left(\psi^{\prime}, \widetilde{U}\right), \\
b_{t}^{\prime}-N^{2} \psi_{y}^{\prime}=-J\left(\psi^{\prime}, \widetilde{B}\right), \\
\psi_{z z t}^{\prime}-\beta y u_{z}^{\prime}+b_{y}^{\prime}=-J\left(\psi^{\prime}, \widetilde{\Psi}_{z z}\right) .
\end{gathered}
$$



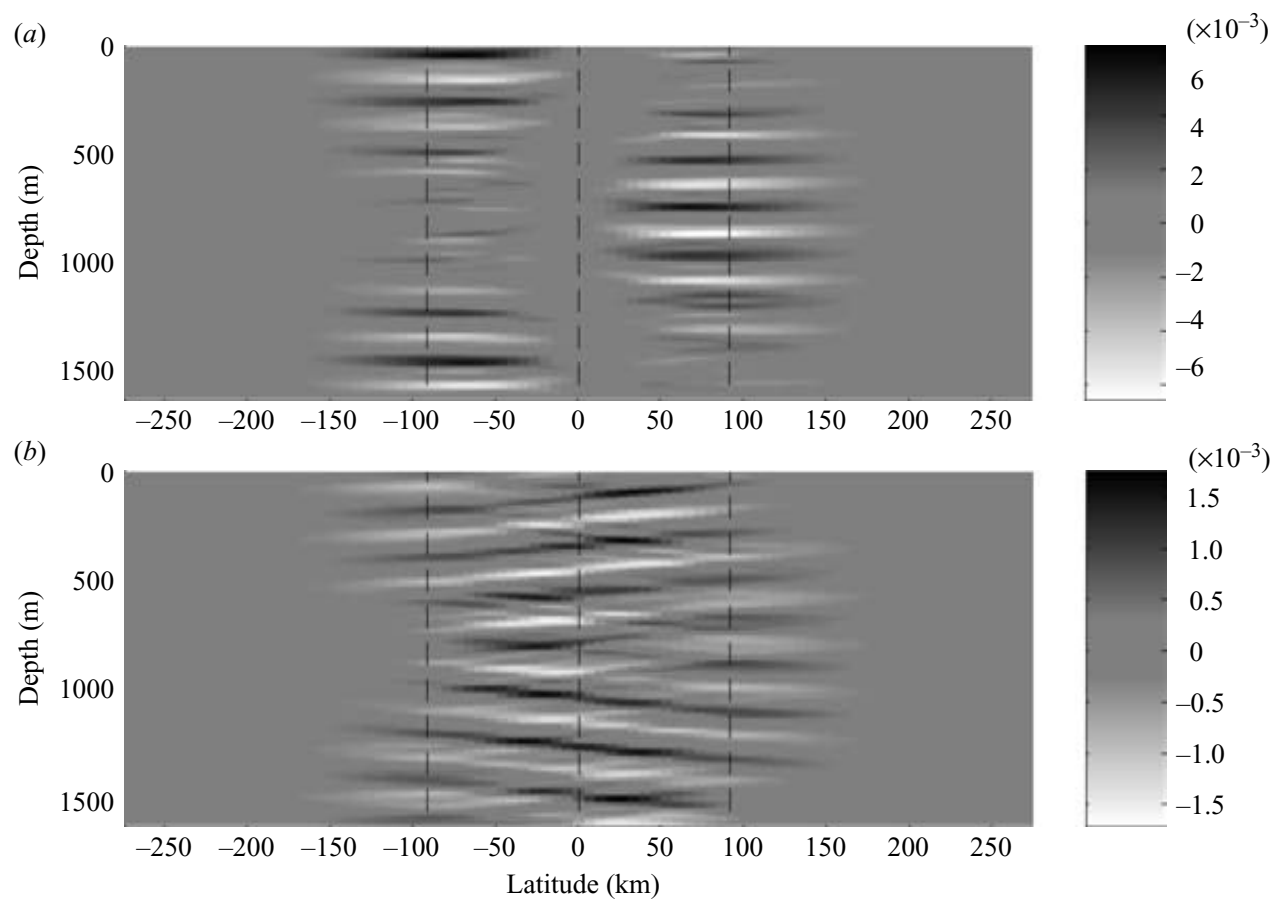

FiguRE 8. Same as figure 5 for the truncated linearized system, except that the vertical $m_{0}$ wavenumber has not been filtered out.

Equations (4.9)-(4.11) are integrated forward in time with small initial random perturbations. An exponential growth is observed. The growing solution of the system is plotted in figure 8 and is very much like the solution obtained for the nonlinear initial-value simulation (figure 5). The vertical mode spectra of the solution at different instants presented in figure $9(a)$ exhibit a sharp peak centred around vertical modes $m / m_{0}=7$ and 8 . Mode $m / m_{0}=7$ corresponds to a meridional wavenumber $n=1$, while vertical mode $m / m_{0}=8$ has a meridional wavenumber $n=0$. The frequency spectra of both vertical modes shown in figure $9(b)$ confirm that relation (4.5) is verified and thus that a parametric resonance takes place involving different meriodional wavenumbers of two distinct vertical modes.

System (4.9)-(4.11) can be reduced to a single, so-called Eliassen-Sawyer equation for $\psi^{\prime}$, by eliminating $u^{\prime}$ and $b^{\prime}$. Performing a scale analysis (see Appendix B), using a non-dimensionalization based on the spatial and time scales of the perturbation, the relevant Eliassen-Sawyer equation for the parametric destabilization of the basic-state MRG wave amounts dimensionally to

$$
\psi_{z z t t}^{\prime}+f^{2} \psi_{z z}^{\prime}+N^{2} \psi_{y y}^{\prime}-f \widetilde{U}_{y} \psi_{z z}^{\prime}+\widetilde{B}_{y} \psi_{y z}^{\prime}=0 .
$$

Comparing (4.12) with (3.4) shows that for a vertically variable shear flow, such as the basic-state MRG wave, besides the term involving lateral shear $\left(f \widetilde{U}_{y}\right)$, the resonance involves the additional contribution of another component of the flow $\left(\widetilde{B}_{y}\right)$.

A multiple-scale analysis of (4.12) is detailed in Appendix B, taking into account the $\left(\cos \left(m_{0} z\right), \sin \left(m_{0} z\right)\right)$ vertical dependence of $f \widetilde{U}_{y}$ and $\widetilde{B}_{y}$. It is shown that the subharmonic resonance reaches its maximum growth rate for the pair of wavenumbers $n=1, m=7 m_{0}$ and $n=0, m=8 m_{0}$ in the case of a Froude number $\alpha>0.084$. It has 
(a)

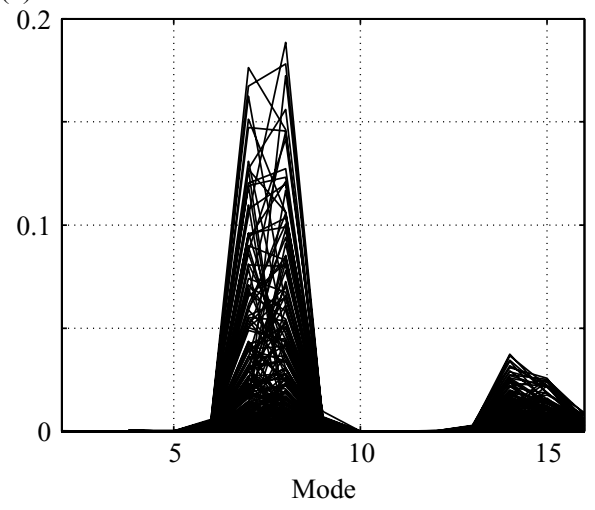

(b)

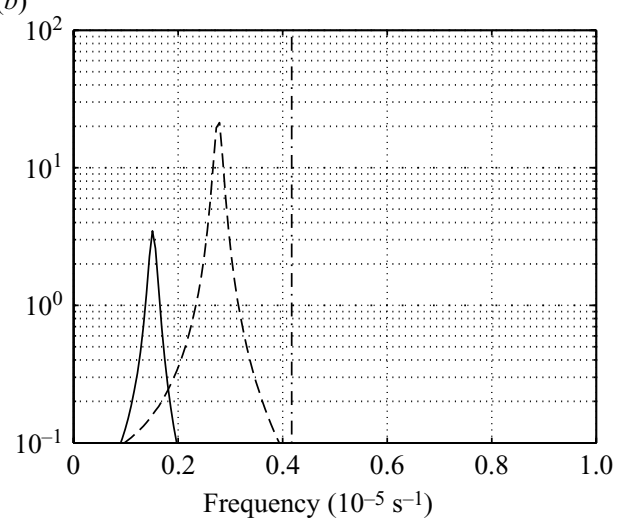

FIGURE 9. Same as figure 6, but for the truncated linearized system.

been established that such resonances of modes 7 and 8 involve a critical Froude number $\alpha_{c} \approx 0.03$, which has been checked numerically to provide a lower bound for the excitation in the truncated linearized system simulation as well as in the initial-value problem of the previous section.

Appendix B proves that (i) the breaking of symmetry with respect to the equator of the MRG wave, through terms $f \widetilde{U}_{y}$ and $\widetilde{B}_{y}$, allows the resonance of distinct meridional modes $n$ and $n+1$ as for the barotropic shear case (\$3.1), and that (ii) the vertical dependence of the MRG wave implies, however, a resonance between distinct vertical modes in contrast to $\$ 3.1$. Moreover, an estimated growth rate for Froude number $\alpha>0.084$ is $\mu \sim \gamma / 8$ as in $\S 3.1$, and differs by less than $20 \%$ from that found in the numerical simulations of the truncated linearized system.

\section{Angular momentum redistribution}

We recall that one of the main motivations of this study mentioned in $\S 1$ is the possible impact of parametric resonance on angular momentum redistribution.

Section 5.2 discusses the implication of the destabilization of the MRG wave on angular momentum, but prior to that, we shall recall the most important results of nonlinear inertial instability that are necessary for its intercomparison with inertialparametric instability.

\subsection{Nonlinear inertial instability: angular momentum homogenization}

Equations (3.1)-(3.3) (where $\omega_{0}$ is set to 0 ) are integrated with the numerical model of Hua et al. (1997). Dissipation terms in (3.1)-(3.3) are $D=v_{z}\left(\partial^{2} / \partial z^{2}\right)+v_{4 h}\left(\partial^{4} / \partial y^{4}\right)$ with $v_{z}=10^{-5} \mathrm{~m}^{2} \mathrm{~s}^{-1}$ and $v_{4 h}=1 \times 10^{8} \mathrm{~m}^{4} \mathrm{~s}^{-1}$ for the numerical stability of the model. Spatial scales $L_{y}=O(100 \mathrm{~km})$ and $H_{z}=O(100 \mathrm{~m})$, require a very anisotropic grid and therefore a hydrostatic assumption. The grid size is $d y=3 \mathrm{~km}$ and $d z=2.5 \mathrm{~m}$. The domain of integration is periodic in the vertical and symmetric about the equator. The constant stratification background is $N=2 \times 10^{-3} \mathrm{~s}^{-1}$.

Nonlinear interactions of baroclinic perturbations lead to a barotropic signal

$$
\frac{\partial}{\partial t} \overline{u^{\prime}}=-\frac{\partial}{\partial y} \overline{u^{\prime} v^{\prime}}
$$



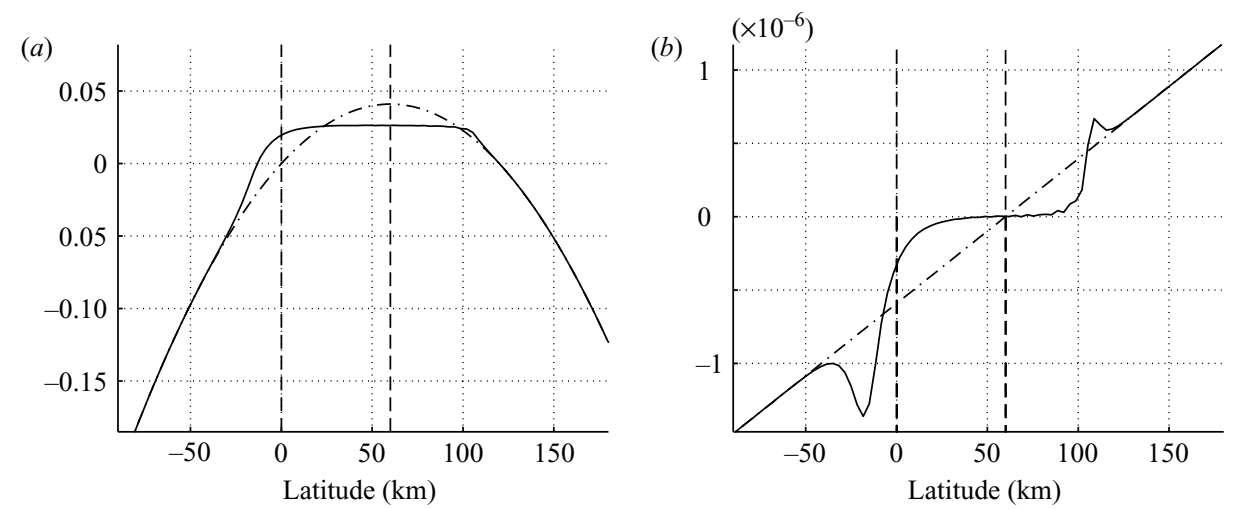

Figure 10. (a) Angular momentum $\bar{M}$. (b) Potential vorticity $\bar{Q}$ (continuous lines for fields at time $t$, dash-dotted line for initial fields). Vertical lines delimit the linearly unstable band $y=0$ and $y=2 y_{0}=\gamma / \beta$.

(obtained by the vertical average of (3.1) and where $\bar{R}$ denotes a vertical average of $R$ ) that will restabilize the system (Griffiths 2003a) in the absence of lateral dissipation of angular momentum.

The final-state barotropic angular momentum distribution (figure $10 a$, solid line) has been redistributed laterally by the baroclinic perturbations

$$
\frac{\partial}{\partial t} \bar{M}=-\frac{\partial}{\partial y} \overline{M v^{\prime}},
$$

(where $M=\gamma y+u^{\prime}-\beta y^{2} / 2$ ) in order to wipe out the initial extra-equatorial angular momentum maximum (dashed line) and thus restabilizing the system with respect to inertial instability. This will also reflect in the vertically integrated potential vorticity distribution $\bar{Q}=\overline{\nabla M \times \nabla \rho}$ which is homogenized to zero inside the unstable latitude band (figure $10 b$ ).

For a large enough supercriticality of the barotropic steady shear $\gamma=\widetilde{U}_{y}$, such that $\gamma / \gamma_{c}>1.19$, where $\gamma_{c}$ is the critical shear value set by vertical viscosity/diffusion (Dunkerton 1981), the nonlinear response will reach large amplitude and occurrences of density overturning by large-scale meridional cells of small vertical wavelength lead to a layering regime (see figure 11 and Appendix A). Such a limit is close to the limit $\left(\gamma / \gamma_{c} \approx 1.16\right)$ found by Griffiths (2003a) when the local Richardson number verifies $R i \approx 1 / 4$, whereas Majda \& Shefter (1998b) show that, for an appropriate definition of the Richardson number, $R i<1 / 4$ corresponds to a flow that overturns. In such regime, density is vertically homogenized over layers of small vertical extent that are separated by sharp jumps in stratification which can reach a very large latitudinal extension.

For further details on nonlinear inertial instability, see Hua et al. (1997) and Griffiths $(2003 a, b)$.

\subsection{Parametric adjustment of the mixed Rossby-gravity wave: angular momentum budgets}

Let us now examine the angular momentum budget in the nonlinear initial-value simulation of $\S 4.1$. As mentioned above, the simulation corresponds to the free nonlinear adjustment of an MRG wave in the absence of any external forcing, so that fluid parcels conserve angular momentum at each instant

$$
M(y, z, t)=u-\frac{1}{2} \beta y^{2},
$$




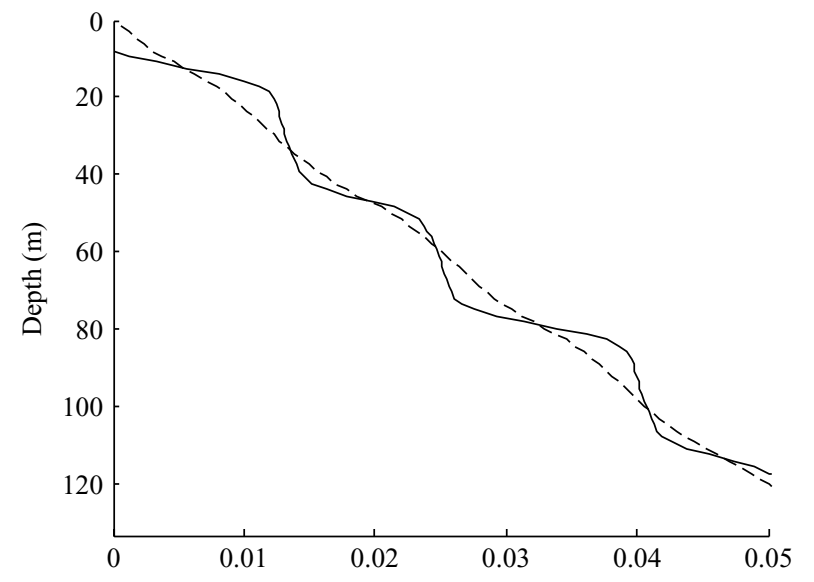

FIGURE 11. Density profile at the dynamical equator $y_{0}=\gamma / 2 \beta$ (solid line) and meridional mean density profile (dashed line).

(since $u$ denotes the total zonal velocity it includes the initialized MRG wave signal)

$$
\frac{\mathrm{d}}{\mathrm{d} t} M=0
$$

The initial profiles of total angular momentum $M$ at $t=0$ with $u=\widetilde{U}$ is plotted in figure $12(a)$. The barotropic component of angular momentum $\bar{M}(y, t=0)$ coincides exactly with the planetary component $-\beta y^{2} / 2$ initially since the MRG wave zonal velocity integrates to zero over a vertical wavelength. Figure 12(a) also shows angular momentum at two depths where the MRG zonal velocity amplitude has opposite phases (dash-dotted lines): the non-zero zonal shear of the MRG wave implies the presence of extrema of angular momentum away from the equator $(\approx 40 \mathrm{~km})$. Figure $12(b)$ shows the corresponding meridional profiles of initial potential vorticity (vertically averaged and at the above-mentioned two depths), which all correspond to the planetary potential vorticity since $v=0$ in our initial conditions. Although the classical inertial instability condition $f Q<0$ is not verified initially, there are meridional maxima of angular momentum displaced from the equator at some depths. (After the initialization and because of the time-oscillation of the wave, these meridional maxima of angular momentum vary in latitude and time as well as the potential vorticity.)

Figure 12(c) shows the meridional profiles of total angular momentum after nonlinear adjustments: there is a partial homogenization of total angular momentum at both vertical levels as well as of barotropic angular momentum. Figure $12(d)$ shows the corresponding meridional profiles of potential vorticity after nonlinear adjustment at the above-mentioned two depths and for its barotropic component. There is also a tendency for a lateral homogenization of potential vorticity symmetrically about the equator. These last two figures should be compared with figures $10(a)$ and $10(b)$ for the case of steady inertial instability. The nonlinear evolution of the parametrically excited motions induced by the basic state MRG wave described in $\S 4.1$ has wiped out the baroclinic extra-equatorial maxima of angular momentum, that are present initially.

More importantly, the barotropic component of angular momentum $\bar{M}(y, t)$ (solid line in figure $12 c$ ) which is now clearly distinct from the planetary contribution, is also 
(a)

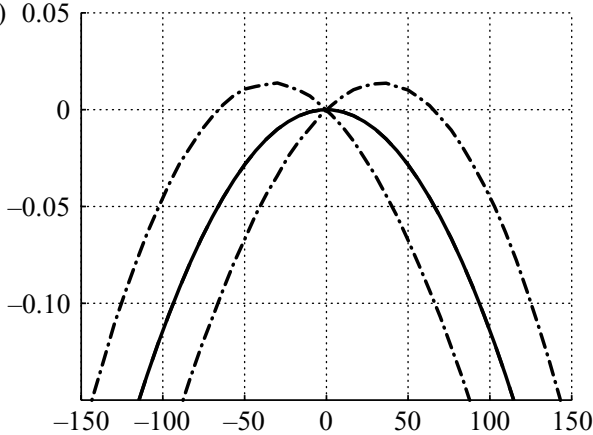

(c)

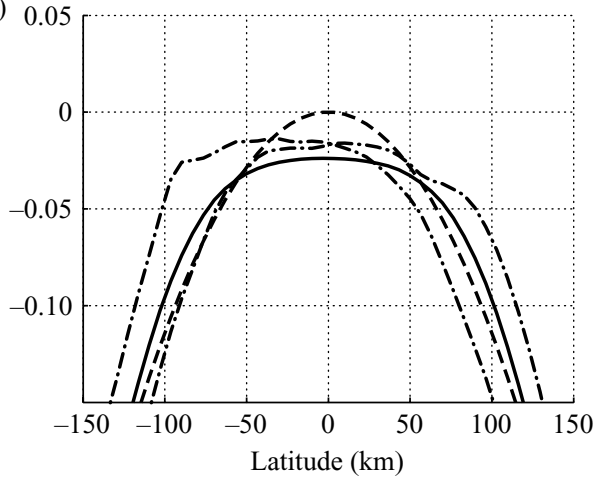

$\left(\times 10^{-6}\right)$

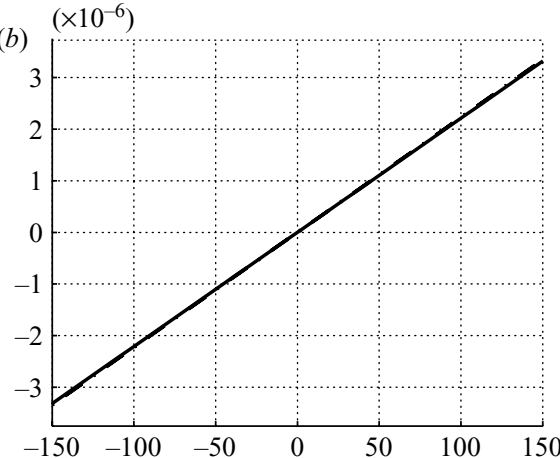

(d) $\left(\times 10^{-6}\right)$

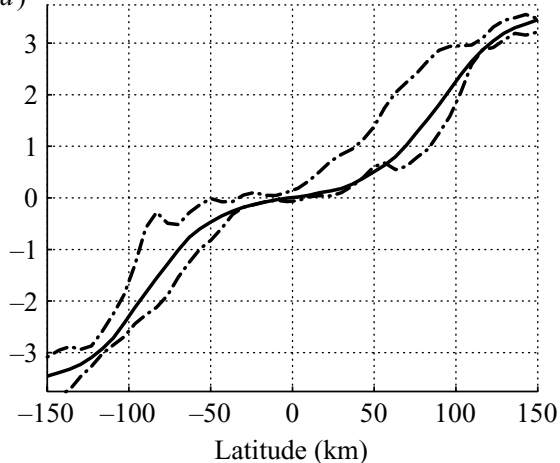

FiguRE 12. MRG wave initial-value problem nonlinear simulation. $(a, c)$ Profiles of angular momentum as a function of latitude: $(a)$ initial time; $(c)$ final time. Dash-dotted curves correspond to the total angular momentum $M$ at two different depths where the zonal velocity amplitude of the MRG wave has opposite phases. Dashed curve: planetary angular momentum contribution. Solid curve: total barotropic angular momentum $\bar{M} .(b, d)$ Profiles of potential vorticity $Q$ as a function of latitude; $(b)$ initial time; $(d)$ final time. Solid curve: vertically averaged potential vorticity. Dash-dotted curves: potential vorticity at the above-mentioned two depths.

homogenized laterally and is symmetric about the equator (while homogenization occurs only in a single hemisphere for inertial instability induced by a steady shear). We emphasize that such barotropic homogenization of angular momentum, symmetric about the equator, necessarily implies westward flow in an equatorial band and extra-equatorial eastward flow (at $y \approx 100 \mathrm{~km}$, figure 13) to globally conserve angular momentum. Since westward flow is often observed at large depths beneath the equatorial thermocline, such nonlinear adjustment of a finite-amplitude MRG wave could thus be relevant for explaining the equatorial oceanic observations. We have checked numerically that the relative amplitude of eastward and westward flows depends on the initial MRG wave's Froude number. However, the latitudinal positions of the eastward maxima always coincide with the critical inertial latitudes $\left(y_{c}= \pm \omega_{0} / 2 \beta\right)$ and are thus independant of the Froude number.

All the above results further support the analogy between the so-called inertialparametric instability and classical inertial instability, since the parametric case presents a tendency for a homogenization of angular momentum and potential vorticity.

Moreover, for a large enough initial Froude number of the MRG wave, the excited modes 7 and 8 which are plotted in figure 5 at their maximum amplitude, 

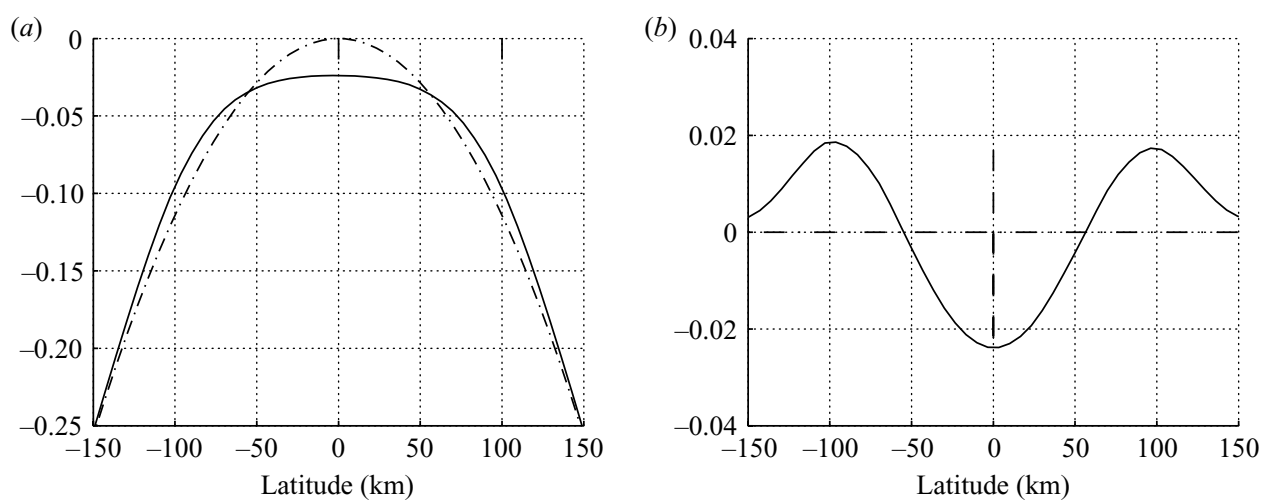

FIGURE 13. (a) Barotropic angular momentum and $(b)$ barotropic zonal velocity profile after nonlinear adjustment of the MRG wave.

subsequently break. This leads to density overturning and thus to the formation of density layering, over large meridional distances. This is similar to what happens in the steady inertial instability problem (\$ 5.1). Such layering regime could be relevant to oceanic observations in the equatorial oceans where meridionally extended layering of salt and temperature have been observed in the upper layers (Richards \& Banks 2002) as well as in deeper regions (d'Orgeville et al. 2004) on both sides of the equator.

It should be pointed out that the breaking of modes 7 and 8 leads to an irreversible mixing of potential vorticity: the energy loss by the MRG wave benefits not only modes 7 and 8 but also the barotropic mode, as well as small meridional and vertical scales through dissipation after breaking has occurred (figure 7). This dissipative character of the breaking process entails the irreversibility of the potential vorticity mixing. Such a mixing occurs on a time scale linked to the breaking of modes 7 and 8 and thus faster than the instability growth rate (figure $7 b$ ).

\section{Summary and discussion}

We have demonstrated the existence of a linear parametric instability triggered by an oscillating shear flow on an equatorial $\beta$-plane under zonally symmetric conditions and for a stratified fluid. This has been shown analytically for the limit of a weak shear amplitude relatively to the oscillating shear frequency. Spatial properties of this instability are solely governed by its forcing frequency $\omega_{0}$. The 'driving' of this instability originates from a specific property of the equatorial $\beta$-plane: the continuous distribution of inertial oscillation frequencies implies that for any given $\omega_{0}$, the periodic shear forcing will excite a subharmonic parametric resonance of inertial oscillations at a critical inertial latitude $y_{c}$ such that

$$
\beta y_{c}= \pm \frac{1}{2} \omega_{0}
$$

(as demonstrated for the homogeneous fluid case). A first consequence is that the faster the shear oscillation, the wider the equatorial band with resonant growth. Stratification comes into play mainly for selecting a most unstable high vertical mode

$$
m_{c} \approx 7.45 m_{0}, \quad m_{0}=\frac{\beta N}{\omega_{0}^{2}},
$$

$m_{0}$ being the equatorial vertical mode which is characteristic of frequency $\omega_{0}$, with a combination resonance of the summed type involving two free meridional modes 
$n=0$ and $n=1$. High vertical free modes are selected by subharmonic parametric instability because they have lower frequencies.

We propose to name such resonant instability an equatorial inertial-parametric instability because (i) of its overall similarity with the classical steady inertial instability triggered by non-zero shear at the equator and (ii) it corresponds to the resonant excitation of free inertio-gravity waves of the equatorial $\beta$-plane.

This inertial-parametric instability is endowed with more physical properties than classical inviscid inertial instability owing to the existence of non-zero steady shear at the equator. In particular, it has a well-defined inviscid linear vertical scale selection, that corresponds to the lowest vertical unstable mode. For a fixed viscosity/diffusion level, linear inertial-parametric instability requires a lower threshold of shear amplitude for the instability to set in than for steady inertial instability, the critical vertical wavenumber being furthermore independent of viscosity. The inertial instability limit is recovered for the regime of $\omega_{0} \ll \gamma$ where inertial instability takes place at a given level on each side of the equator for each half-period of the shear flow oscillation.

The previous idealized study requires an external forcing that maintains the oscillating shear flow. In a geophysical context, such oscillating shear can be attributed to large-vertical-scale free equatorial waves that are ubiquitous in atmospheric and oceanic observations and do not require an external forcing. We have in that context studied the nonlinear adjustment of a zonally symmetric mixed Rossby-gravity (MRG) wave, which is the simplest zonally symmetric wave with non-zero zonal shear at the equator. Since it is well known that linear equatorial waves are not exact solutions of the nonlinear equations of motion for the equatorial $\beta$-plane case, initialvalue simulations of such MRG waves with moderate basic-state Froude numbers $0.03<\alpha<0.4$, in the absence of any external forcing, have been performed. They show that the nonlinear adjustment process involves a parametric resonant instability of the inertial-parametric type. The final nonlinearly equilibrated signal is dominated by two high vertical modes, free inertio-gravity waves, with vertical scales 7 and 8 times smaller than the initial basic $\mathrm{MRG}$ wave, and with meridional wavenumbers 1 and 0 , respectively.

We have proved that such a resonant response could be predicted by a truncated linearization of the equations of motions about the basic MRG wave state, where forcing terms due to the advection of the perturbation by the MRG wave which cannot induce energy tranfer have been discarded. The truncated system amounts to only keeping the advection by the perturbation of the MRG wave. More fundamentally, the symmetry breaking enabled by the antisymmetric shear and meridional buoyancy gradient of the MRG wave play a fundamental role in the development of the instability.

In our opinion, one geophysically relevant implication of such inertial-parametric instability of the MRG wave concerns its consequences on angular momentum redistribution. The nonlinear adjustment process of the MRG wave exhibits over the whole domain height a substantial meridional homogenization of angular momentum and of potential vorticity symmetrically about the equator on average in the vertical. The nonlinear saturation of the inertial-parametric instability induced by laterally and vertically sheared flows behaves analogously to the classical inertial instability triggered by a steady shear flow. The nonlinear evolution of both instabilities favours a meridional homogenization of angular momentum and potential vorticity. This mixing of potential vorticity is irreversible since it involves dissipative processes through the breaking of the unstable modes for high enough supercriticalities of the basic state. 
However, the main difference between the two types of instability is that steady inertial instability homogenizes only on a given side of the equator, whereas inertialparametric instability excited by the MRG wave yields a homogenization which is symmetric about the equator. Such angular momentum meridional redistribution developed by an MRG wave creates westward flow at the equator and eastward extra-equatorial flow, which is often observed in deep oceanic equatorial flows.

Another implication of such angular momentum redistribution by large vertical and extended meridional circulation cells is the mixing of all properties conserved by fluid parcels. Such lateral mixing has been already noted by Richards \& Edwards (2003) for the case of steady inertial instability. Since in a stratified medium density is one particular conserved property, this inertial-parametric instability is able to create density layering for shear amplitudes large enough to cause local density overturning. This process is akin to the one put forward by Majda \& Shefter $(1998 a, b)$ with a resonant excitation of internal waves in a stratified medium. It should be pointed out that in that case $\omega_{0} \approx N / 2$, whereas for equatorial inertial-parametric instability, $\omega_{0} \ll N / 2$. The layering which is thus produced is associated with low meridional wavenumber inertio-gravity waves which have a large meridional scale extent in contrast to internal waves motions. Such a layering mechanism induced on both sides of the equator by an equatorial oscillating shear flow is proposed by d'Orgeville et al. (2005) to explain the layering observed at depths in the equatorial Atlantic over considerable meridional $(200 \mathrm{~km})$ and zonal extent $(800 \mathrm{~km})$.

In this work, we have focused on the irreversible mixing of potential vorticity brought about by wave breaking in the meridional $(y, z)$-plane of high vertical modes that are excited by inertial-parametric instability. Such meridional plane mixing is consistent with our zonally symmetric assumption. Bouchut, Le Sommer \& Zeitlin (2004) study instead the irreversible mixing of potential vorticity induced by wave breaking in a one-layer shallow-water model, where the zonal pressure gradient plays a central role in the wave steepening. It could be of interest for further studies to take into account both types of breaking mechanism.

The authors would like to thank Drs F. Marin and R. Schopp and Mr G. Forget for valuable comments on this work. Numerous thoughtful comments by referees are greatfully acknowledged. Support from the Institut du Développement et des Ressources en Informatique Scientifique via grant. 41299 is acknowledged.

\section{Appendix A. Escaping Hide's theorem: effect of lateral dissipation on persistent layering}

The zonal equation of motion for a zonally symmetric flow

$$
\frac{\mathrm{d} u}{\mathrm{~d} t}-\beta y v=D_{u}
$$

where $D_{u}$ is dissipation, is rewritten in terms of angular momentum as $M=u-\beta y^{2} / 2$

$$
\frac{\mathrm{d} M}{\mathrm{~d} t}=D_{u} .
$$

In the above equations, $u$ denotes the total zonal velocity. Such an equation states angular momentum conservation by fluid parcels except for dissipation of zonal velocity. 
Vertical dissipation acting alone : Hide's theorem

For a downgradient vertical dissipation, e.g. $D_{u}=v_{z}\left(\partial^{2} u / \partial z^{2}\right)$, equation (A 1) becomes

$$
\frac{\mathrm{d} M}{\mathrm{~d} t}=v_{z} \frac{\partial^{2} M}{\partial z^{2}},
$$

and Hide's theorem applies (e.g. Held \& Hou 1980): for any amount of dissipation $v_{z}$, even for vanishingly small values, a steady solution of (A 2) cannot present a vertically localized extremum of angular momentum except at a boundary, since at each point such solution verifies the flux equation

$$
\nabla \cdot(M \mathbf{V})=v_{z} \frac{\partial^{2} M}{\partial z^{2}} .
$$

Hide's theorem applies on the equatorial $\beta$-plane since vertical variations of $M$ exactly coincide with those of the zonal velocity $u$.

Laplacian lateral dissipation: escaping Hide's theorem

In the case of both a vertical and lateral downgradient dissipation of zonal velocity $u$, the applicability of Hide's theorem can depend on the explicit choice of lateral dissipation.

For a Laplacian lateral dissipation $\left(D_{u}=v_{z}\left(\partial^{2} M / \partial z^{2}\right)+\kappa_{h}\left(\partial^{2} u / \partial y^{2}\right)\right)$ since $D_{u} \neq D_{M}$, a flux form equation for angular momentum cannot apply because of the term in $y^{2}$ in planetary angular momentum $-\beta y^{2} / 2$. Hide's theorem does not apply.

\section{Persistent layering}

For the numerical simulations of $\S 5.1$, if lateral Laplacian diffusion/dissipation are used, we have found that a baroclinic meridional circulation is present in the final state, enabling the existence of extrema in the vertical of angular momentum as well as a persistent layering. These results provide an example that escapes the conditions necessary for Hide's theorem to apply. In such a case, the barotropic flow verifies

$$
\frac{\partial}{\partial y} \overline{M v}=\kappa_{h} \frac{\partial^{2} \bar{u}}{\partial y^{2}} .
$$

\section{Appendix B. Parametric instability of the geostrophic component of the mixed Rossby-gravity wave}

In this Appendix, we shall analytically (i) justify the resonant instability between two distinct vertical modes occurring in the MRG wave case (§4), (ii) provide an estimate of the growth rate, and (iii) establish the role played by the Froude number of the basic state. In $\S$ B.1, system (4.9)-(4.11) is reduced to a simplified EliassenSawyer equation relevant for high vertical modes. In $\S$ B.2, a multiple scale analysis is performed.

To alleviate notations, the prime sign denoting perturbation is omitted in this Appendix and we shall denote partial derivatives with a subscript in $\S$ B.1.

\section{B.1. Relevant Eliassen-Sawyer equation}

System (4.9)-(4.11) can be reduced to a scalar Eliassen-Sawyer equation for $\psi$ by eliminating $u$ and $b$

$$
\begin{aligned}
\psi_{t t z z} & +f^{2} \psi_{z z}+N^{2} \psi_{y y}=\left(\widetilde{\Psi}_{z z y} \psi_{z}-\widetilde{\Psi}_{z z z} \psi_{y}\right)_{t} \\
& +\left(f \widetilde{U}_{y z}-\widetilde{B}_{y y}\right) \psi_{z}-\left(\widetilde{B}_{y}+f \widetilde{U}_{z}\right) \psi_{y z} \\
& +f \widetilde{U}_{y} \psi_{z z}+\widetilde{B}_{z} \psi_{y y}+\left(\widetilde{B}_{z y}-f \widetilde{U}_{z z}\right) \psi_{y}
\end{aligned}
$$


To simplify the above equation we shall restrict ourselves to the case of a high vertical mode $m$ and introduce the small parameter

$$
\epsilon=\sqrt{\frac{m_{0}}{m}}
$$

Equation (B 1) is non-dimensionalized for this vertical mode $m$ with the same scaling as in the beginning of $\S 3.1$ (and asterisks are omitted hereinafter)

$$
\begin{aligned}
\psi_{t t z z} & +y^{2} \psi_{z z}+\psi_{y y}=\left(m V_{0} / N\right) \epsilon e^{-\epsilon^{2} y^{2} / 2}\left[-\epsilon^{3}\left\{\cos (t / \epsilon)\left(y \sin \left(\epsilon^{2} z\right) \psi_{z}+\cos \left(\epsilon^{2} z\right) \psi_{y}\right)\right\}_{t}\right. \\
& +\sin (t / \epsilon) \sin \left(\epsilon^{2} z\right)\left\{\left(-\left[\epsilon^{4} y^{3}-\epsilon^{2} y\right]+\left[3 \epsilon^{2} y-\epsilon^{4} y^{3}\right]\right) \psi_{z}-\left(\left[1-\epsilon^{2} y^{2}\right]-\epsilon^{2} y^{2}\right) \psi_{z y}\right\} \\
& \left.+\sin (t / \epsilon) \cos \left(\epsilon^{2} z\right)\left\{\left(y-\epsilon^{2} y^{3}\right) \psi_{z z}+\epsilon^{2} y \psi_{y y}+\left(\left[\epsilon^{2}-\epsilon^{4} y^{2}\right]+\epsilon^{4} y^{2}\right) \psi_{y}\right\}\right] .
\end{aligned}
$$

Assuming $\epsilon \ll 1$, a singular perturbation expansion in $y$ gives the relevant nondimensional Eliassen-Sawyer equation

$$
\psi_{z z t t}+y^{2} \psi_{z z}+\psi_{y y}=\frac{m V_{0}}{N} \epsilon \sin (t / \epsilon)\left(y \psi_{z z} \cos \left(\epsilon^{2} z\right)-\psi_{y z} \sin \left(\epsilon^{2} z\right)\right),
$$

since all other terms in the original equation (B 1) are of order $\epsilon^{2}$ smaller than terms kept in (B 2). This scale analysis is valid when $y=O(1)$, which can be justified by the exponential decay of the projecting Hermite mode in $\S$ B.2.

Finally, the dimensional Eliassen-Sawyer equation, relevant for high vertical mode perturbations, is

$$
\psi_{t t z z}+\beta^{2} y^{2} \psi_{z z}+N^{2} \psi_{y y}-\frac{V_{0}}{\lambda_{0}} \sin \left(\omega_{0} t\right)\left[\beta y \cos \left(m_{0} z\right) \psi_{z z}-N \sin \left(m_{0} z\right) \psi_{y z}\right]=0,
$$

which corresponds to

$$
\psi_{t t z z}+f^{2} \psi_{z z}+N^{2} \psi_{y y}-f \widetilde{U}_{y} \psi_{z z}+\widetilde{B}_{y} \psi_{y z}=0
$$

B.2. Multiple-scale analysis

In order to work with non-dimensional quantities which only depend on the basicstate MRG wave and not on the vertical scale of the perturbations, the truncated Eliassen-Sawyer equation (B 3) is non-dimensionalized with the characteristic scales of the MRG wave $\left(z^{*}=m_{0} z, y^{*}=y / \lambda_{0}, t^{*}=t \omega_{0}\right.$, and asterisks are omitted hereinafter $)$

$$
\frac{\partial^{4} \psi}{\partial t^{2} \partial z^{2}}+y^{2} \frac{\partial^{2} \psi}{\partial z^{2}}+\frac{\partial^{2} \psi}{\partial y^{2}}=\alpha \sin (t)\left(y \frac{\partial^{2} \psi}{\partial z^{2}} \cos (z)-\frac{\partial^{2} \psi}{\partial y \partial z} \sin (z)\right) .
$$

The key parameter is the amplitude of the MRG wave through the Froude number $\alpha$ defined in (4.4).

Equation (B 5) is projected on the basis of meridional/vertical mode $\{l, k\}$ such that

$$
\psi(y, z, t)=\sum_{k \geqslant 1} \sum_{l \geqslant 0} \varphi_{l, k}(y) \phi_{l, k}(t) \sin \left(k z+\theta_{l, k}\right),
$$

with $\varphi_{l, k}(y)=H_{l}(\sqrt{k} y) \exp \left(-k y^{2} / 2\right)$. Making use of the recurrence relations of Hermite functions for a given vertical mode $k$

$$
\begin{aligned}
y \varphi_{l, k} & =\sqrt{\frac{1}{k}}\left(l \varphi_{l-1, k}+\frac{1}{2} \varphi_{l+1, k}\right), \\
\frac{\partial}{\partial y} \varphi_{l, k} & =\sqrt{k}\left(l \varphi_{l-1, k}-\frac{1}{2} \varphi_{l+1, k}\right),
\end{aligned}
$$




$$
\frac{\partial^{2}}{\partial y^{2}} \varphi_{l, k}=\left(k(2 l+1)-(k y)^{2}\right) \varphi_{l, k},
$$

and projecting on a given vertical mode $p$ and its meridional mode $n$, this yields

$$
\begin{aligned}
& \frac{\partial^{2}}{\partial t^{2}} \phi_{n, p}+\delta_{n, p}^{2} \phi_{n, p}=\frac{\alpha}{\sqrt{p}} \sin (t) \\
& \quad \times\left(\frac{1}{2} \cos \left(\theta_{n, p}-\theta_{n-1, p+1}\right) \phi_{n-1, p+1}+(n+1) \cos \left(\theta_{n, p}-\theta_{n+1, p-1}\right) \phi_{n+1, p-1}\right),
\end{aligned}
$$

where $\delta_{n, p}^{2}=(2 n+1) / p$. Note that we have used the approximation that Hermite functions of distinct vertical modes $k$ and $k+1$ are orthonormal.

The oscillation with height of the basic state thus involves possible resonant interactions between distinct vertical modes, such that they correspond to $\{n, p+1\}$ and $\{n+1, p\}$, or for a dimensional vertical wavenumber to $\left\{n, m+m_{0}\right\}$ and $\{n+1, m\}$. Combination resonances of the summed type will occur for appropriate values of $\theta_{n+1, p}-\theta_{n, p+1}$, the optimal case being $\theta_{n+1, p}-\theta_{n, p+1}=0$ or $\pi$. In the latter case, the multiple-scale analysis of (B 6) with $\alpha \ll 1$ and $\tau=\alpha t$ is analogous to that used in $\S 3$

$$
\phi_{n, p}(t, \tau)=A_{n, p}^{0}(t, \tau)+\alpha A_{n, p}^{1}(t, \tau)+O\left(\alpha^{2}\right) .
$$

At order 0 ,

At order 1,

$$
A_{n, p}^{0}(t, \tau)=\mathrm{B}_{n, p}(\tau) \exp \left(\mathrm{i} \delta_{n, p} t\right)+\text { c.c. }
$$

$$
\frac{\partial^{2} A_{n, p}^{1}}{\partial t^{2}}+\delta_{n, p}^{2} A_{n, p}^{1}=-\left(2 \frac{\partial^{2} A_{n, p}^{0}}{\partial \tau \partial t}\right)+\frac{1}{\sqrt{p}}\left(\frac{1}{2} A_{n-1, p+1}^{0}+(n+1) A_{n+1, p-1}^{0}\right) \sin (t) .
$$

Proceeding as in $\S 3.1$, the instability condition is found to be

$$
\alpha^{2} \frac{(n+1)}{8 \sqrt{(2 n+1)(2 n+3)}}>\left(1-\sqrt{\frac{2 n+1}{p+1}}-\sqrt{\frac{2 n+3}{p}}\right)^{2} .
$$

A countable infinite number of pairs $\{n, p+1\}$ and $\{n+1, p\}$ can be found to be unstable for a given Froude number $\alpha$. For such a pair, the dimensional growth rate is

$$
\mu=\frac{1}{2} \gamma \sqrt{\frac{(n+1)}{8 \sqrt{(2 n+1)(2 n+3)}}-\frac{\omega_{0}^{2}}{\gamma^{2}}\left(1-\sqrt{\frac{2 n+1}{p+1}}-\sqrt{\frac{2 n+3}{p}}\right)^{2}} .
$$

This equation has to be compared with (3.6).

In the barotropic shear case $(\S 3)$, the spectrum of unstable vertical modes is continuous and it is possible to find a perfect resonance for a vertical mode $m$ such that the detuning (second term of the radicand in (3.6)) is exactly zero. The growth rate for perfect resonance is $\frac{1}{2} \gamma \sqrt{(n+1) / 8 \sqrt{(2 n+1)(2 n+3)}}$ and leads to an inviscid vertical scale selection for the vertical mode of $m \sim 7.45 m_{0}$ (§3.2).

In the MRG wave case, because the spectrum of unstable vertical modes is discrete ( $p$ necessarily takes integer value), it is no longer possible to find a perfect resonance for which the detuning (second term of the radicand in (B 7)) is zero. However the effect of the detuning on the growth rate amplitude decreases with the Froude number $\alpha=\gamma / \omega_{0}$ and for sufficently large value of $\alpha$ it should be possible to have a vertical scale selection due to the perfect resonance term. 
Indeed we have checked numerically in the range of $0 \leqslant n \leqslant 200$ and $1 \leqslant p \leqslant 1000$ that for $\alpha \geqslant 0.084$ the pair $\{n=0, p=8\}$ and $\{n=1, p=7\}$ has the maximum growth rate. For the MRG wave case, a similar vertical mode selection to the barotropic shear case is thus recovered for large enough Froude number.

The growth rate for the pair $\{n=0, p=8\}$ and $\{n=1, p=7\}$, predicted by (B 7), has been checked to differ by less than $20 \%$ from the one found in the numerical simulations of $\S 4.2$. We suspect that this discrepancy is due to the approximation of singular expansion used in $\S$ B.1.

Furthermore the detuning in (B 7) implies an inviscid critical Froude number

$$
\alpha_{c} \sim 0.03
$$

for the growth of the pair $\{n=0, p=8\}$ and $\{n=1, p=7\}$.

\section{REFERENCES}

Anderson, D. T. \& Gill, A. E. 1979 Beta dispersion of inertial waves. J. Geophys. Res. 84, $1836-1842$.

Benielli, D. \& Sommeria, J. 1998 Excitation and breaking of internal waves by parametric instability. J. Fluid Mech. 374, 117-144.

Bouchut, F., Le Sommer, J. \& Zeitlin, V. 2004 Transport and mixing during the breaking of balanced and unbalanced equatorial waves. Chaos (in press).

Bourlès, B., Andrí́, C., Gouriou, Y., Eldin, G., Du Penhoat, Y., Freudenthal, S., Dewitte, B., Gallois, F., Chuchla, R., Baurand, F., Aman, A. \& Kouadio, G. 2003 The deep currents in the eastern equatorial Atlantic ocean. Geophys. Res. Lett. 30, 8002, doi:10.1029/2002GL015095.

Bunge, L., Provost, C., Lilly, J., D’Orgeville, M., Kartavtseff, A. \& Melice, J.-L. 2005 Variability of the horizontal velocity structure in the upper $1600 \mathrm{~m}$ of the water column on the equator at $10^{\circ} \mathrm{W}$. J. Phys. Oceanogr. (submitted).

Dunkerton, T. J. 1981 On the inertial stability of the equatorial middle atmosphere. J. Atmos. Sci. 38, 2354-2364.

FIRING, E. 1987 Deep zonal currents in the central equatorial Pacific. J. Mar. Res. 45, 791-812.

Gill, A. E. 1982 Atmosphere-Ocean Dynamics. Academic.

Gouriou, Y., Andrié, C., Bourlès, B., Freudenthal, S., Arnault, S., Aman, A., Eldin, G., du Penhoat, Y., Baurand, F., Gallois, F. \& Chuchla, R. 2001 Deep circulation in the equatorial Atlantic ocean. Geophys. Res. Lett. 28, 819-822.

Griffiths, S. D. $2003 a$ The nonlinear evolution of zonally symmetric equatorial inertial instability. J. Fluid Mech. 474, 245-273.

GRIFFITHS, S. D. $2003 b$ Nonlinear vertical scale selection in equatorial inertial instability. J. Atmos. Sci. 60, 977-990.

Held, I. M. \& Hou, A. Y. 1980 Nonlinear axially symmetric circulation in a nearly inviscid atmosphere. J. Atmos. Sci. 37, 515-533.

Hoskins, B. J. 1974 The role of potential vorticity in symmetric stability and instability. Q. J. R. Met. Soc. 100, 480-482.

Hua, B. L., Marin, F. \& Schopp, R. 2003 Three-dimensional dynamics of the subsurface countercurrents and equatorial thermostad. Part I : Formulation of the problem and generic properties. J. Phys. Oceanogr. 33, 2588-2609.

Hua, B. L., Moore, D. W. \& Le Gentil, S. 1997 Inertial nonlinear equilibration of equatorial flows. J. Fluid Mech. 331, 345-371.

Kerswell, R. R. 2002 Elliptical instability. Annu. Rev. Fluid Mech. 34, 86-113.

Leblanc, S. 2003 Internal waves resonances in strain flows. J. Fluid Mech. 477, 259-293.

MCWilliams, J. C. \& YAVNEH, I. 1998 Fluctuation growth and instability associated with a singularity of the balance equations. Phys. Fluids A 10, 2587-2596.

MajdA, A. J. \& Shefter, M. $1998 a$ The instability of stratified flows at large richardson numbers. Proc. Natl Acad. Sci. 95, 7850-7853. 
Majda, A. J. \& Shefter, M. $1998 b$ Elementary stratified flows with instability at large richardson numbers. J. Fluid Mech. 376, 319-350.

Marin, F., Hua, B. L. \& Wacongne, S. 2000 The equatorial thermostad and subsurface countercurrents in the light of the dynamics of atmospheric Hadley cells. J. Mar. Res. 58, 405-437.

Marin, F., Schopp, R. \& HuA, B. 2003 Three-dimensional dynamics of the subsurface countercurrents and equatorial thermostad. Part II: Influence of large-scale ventilation and of equatorial winds. J. Phys. Oceanogr. 33, 2610-2626.

Mu, M., Shepherd, T. G. \& Swanson, K. 1996 On nonlinear symmetric stability and the nonlinear saturation of symmetric instability. J. Atmos. Sci. 53, 2918-2923.

NaYfeh, A. H. \& Mook, D. T. 1979 Nonlinear Oscillations. Wiley Interscience.

D’Orgeville, M., Hua, B. L., Schopp, R. \& Bunge, L. 2004 Extended deep equatorial layering as a possible imprint of inertial instability. Geophys. Res. Lett. 33, L22303.

Pavec, M., Carton, X. \& Swaters, G. 2005 Baroclinic instability of frontal geostrophic currents over a slope. J. Phys. Ocean. (in press).

Pedlosky, J. \& Thomson, J. 2003 Baroclinic instability of time-dependent currents. J. Fluid Mech. 490, 189-215.

Poulin, F., Flierl, G. R. \& Pedlosky, J. 2003 Parametric instability in oscillatory shear flows. J. Fluid Mech. 481, 329-353.

Richards, K. J. \& BANKs, H. 2002 Characteristics of interleaving in the western equatorial Pacific. J. Geophys. Res. 107, (C12), 3231, doi:10.1029/2001JC000971.

Richards, K. J. \& EDWARdS, N. R. 2003 Lateral mixing in the equatorial Pacific: the importance of inertial instability. Geophys. Res. Lett. 30, 1888, doi:10.1029/2003GL017768.

Staquet, C. \& Sommeria, J. 2002 Internal gravity waves: from instability to turbulence. Annu. Rev. Fluid Mech. 34, 559-593.

Stevens, D. E. 1983 On symmetric stability and instability of zonal mean flows near the equator. J. Atmos. Sci. 40, 882-893. 